\title{
EXTENDING INVARIANT COMPLEX STRUCTURES
}

\author{
RUTWIG CAMPOAMOR STURSBERG, ISOLDA E. CARDOSO, AND GABRIELA P. OVANDO
}

\begin{abstract}
We study the problem of extending a complex structure to a given Lie algebra $\mathfrak{g}$, which is firstly defined on an ideal $\mathfrak{h} \subset \mathfrak{g}$. We consider the next situations: $\mathfrak{h}$ is either complex or it is totally real. The next question is to equip $\mathfrak{g}$ with an additional structure, such as a (non)-definite metric or a symplectic structure and to ask either $\mathfrak{h}$ is non-degenerate, isotropic, etc. with respect to this structure, by imposing a compatibility assumption. We show that this implies certain constraints on the algebraic structure of $\mathfrak{g}$. Constructive examples illustrating this situation are shown, in particular computations in dimension six are given.
\end{abstract}

\section{INTRODUCTION}

An important source of complex manifolds is provided by homogeneous manifolds $M=G / H$ with trivial isotropy, that is $H=\{0\}$ so that $M$ is itself a Lie group and the geometric structure is invariant under left-translations. Thus the geometric structure is determined at the Lie algebra level. This setting enables the construction and study of many examples and applications, which in the history (starting by the Erlangen problem by Klein) gave answers to several interesting problems, such as existence of complex, symplectic, pseudo-Kähler, Kähler but non-symplectic structures, as for instance the Kodaira-Thurston manifold).

In dimension four a general classification of Lie groups provided with invariant complex structures is known [29, 32] while the homogeneous case was more recently completed in [14] but no general result is known in higher dimensions. This is an open topic of active research now, as for instance the six-dimensional situation starting by the existence problem of such structures (see 2, 18, 20, 21, 24 for advances in this direction). Indeed the existence and the classification problems become more complicated in higher dimensions. Alternative solutions to this is the consideration of other elements such as an additional geometrical structure related to the (almost) complex structure, giving rise to Hermitian or anti-Hermitian metrics, (pseudo)-Kähler or complex symplectic structures, tamed complex structures, etc.

Our approach here is the extension of complex structures on a given Lie algebra $\mathfrak{g}$. Our motivation and starting point is the following observation: with the exception of the Lie algebras with Heisenberg commutator (see for instance [30]), in dimension four, most of the Lie algebras endowed with a complex structure admit an ideal which is either complex or totally real.

This suggests the study of the relationship between the algebraic structure of $\mathfrak{g}$ and the existence problem of complex structures on $\mathfrak{g}$ in the following frame: determine a complex structure on $\mathfrak{g}$ in such way that a fixed ideal $\mathfrak{h}$ is complex or totally real.

(2000) Mathematics Subject Classification: 53C15, 53C55, 53D05, 22E25, $17 \mathrm{~B} 56$. 
The underlying algebraic situation is the so called extension problem: extend the structure of the Lie algebra $\mathfrak{h}$ to a Lie algebra $\mathfrak{g}$ in such way that $\mathfrak{h} \subseteq \mathfrak{g}$ is an ideal of $\mathfrak{g}$, which gives rise to the following exact sequence of Lie algebras:

$$
0 \rightarrow \mathfrak{h} \rightarrow \mathfrak{g} \rightarrow \mathfrak{g} / \mathfrak{h} \rightarrow 0 .
$$

The extension problem goes back to Chevalley and Eilenberg [13] and it is a current research topic in more general situations (see for instance [1] and references therein).

As we shall see the existence of a complex or a totally real ideal on $\mathfrak{g}$ imposes extra conditions on the algebraic structure of $\mathfrak{g}$. Note that this viewpoint which makes use of algebraic tools was useful in several works (see [8, 2, 10, 22, 23, 25, 26] for instance).

The next step is to add a non-degenerate symmetric or skew-symmetric bilinear map, that is, a metric or a symplectic structure satisfying some compatibility condition with respect to the complex structure, obtaining Hermitian or anti-Hermitian structures (also known as Norden metrics) or pseudo-Kähler or complex symplectic structures. In this case one asks the ideal to be isotropic, non-degenerate, etc. conditions which also impose restrictions on the algebraic structure of $\mathfrak{g}$.

Our main results include:

- the algebraic conditions to extend a complex structure defined on a ideal of a given Lie algebra (in terms of representations of Lie algebras and cohomology);

- the extension of almost Hermitian or almost anti-Hermitian structures starting with an almost Hermitian or almost anti-Hermitian ideal, also symplectic correspondence due to

$\left\{\begin{array}{c}\text { Hermitian structures } \\ \text { such that } \nabla J=0\end{array}\right\} \quad \longleftrightarrow\{$ pseudo-Kähler structures for $J\}$

$\left\{\begin{array}{l}\text { anti-Hermitian structures } \\ \text { such that } \nabla J=0\end{array}\right\} \longleftrightarrow\{$ complex-symplectic structures for $J\}$

where $\nabla$ denotes the Levi-Civita connection.

- Applications in the form of several examples in different situations. In particular computations in dimension six are given providing examples of complex structures on $\mathfrak{g}$ such that the ideal $\mathfrak{h}$ is totally real. In this framework this was possible since the integrability condition for the almost complex structure was reduced to a linear equation which was possible to be solved.

Althought all questions are presented in an algebraic setting, several examples and applications are shown along the paper. In this sense geometrical implications such as curvature, special connections, etc. have been considered by different authors in particular and separate cases (see [3, 4, 7, 15]). In the symplectic case, the study of symplectic Lie groups, with special interest on Lagrangian extensions, was done in [9]. Our proposal here could be go on in this direction.

\section{Preliminaries}

In this section we recall basic facts related to the cohomology of Lie algebras and we introduce complex structures on Lie algebras in this setting. 
2.1. Cohomology of Lie algebras. Let $\left(\mathfrak{k},[\cdot, \cdot]_{\mathfrak{k}}\right)$ and $\left(\mathfrak{h},[\cdot, \cdot]_{\mathfrak{h}}\right)$ be Lie algebras and let $\pi$ denote a representation from $\mathfrak{k}$ into $\mathfrak{h}$ by derivations.

Let $C^{1}(\mathfrak{k}, \pi)$ be the space of linear morphisms of $\mathfrak{k}$ into $\mathfrak{h}$ and for $s>1$ let $C^{s}(\mathfrak{k}, \pi)$ be the space of $s$-alternanting maps of $\mathfrak{k} \times \ldots \times \mathfrak{k}$ (s factors) into $\mathfrak{h}$.

The coboundary operator is a linear operator $d: C^{i}(\mathfrak{k}, \pi) \rightarrow C^{i+1}(\mathfrak{k}, \pi)$ for all $i \geq 1$. If $\theta$ is an element of $C^{1}(\mathfrak{k}, \pi)$ one has

$$
d \theta(x, y)=\pi(x) \theta(y)-\pi(y) \theta(x)-\theta\left([x, y]_{\mathfrak{k}}\right) \quad \text { for all } x, y \in \mathfrak{k}
$$

The map $\theta \rightarrow d \theta$ is linear and the 1-cocycles are the elements of the kernel of $d$, denoted by $Z^{1}(\mathfrak{k}, \pi)$. Let $h \in \mathfrak{h}$ and take $\theta_{h} \in C^{1}(\mathfrak{k}, \pi)$

$$
\theta_{h}(x)=\pi(x) h \quad \text { for } x \in \mathfrak{k} .
$$

It follows from a trivial calculation that $d \theta_{h}=0$ for all $h \in \mathfrak{h}$, hence $\theta_{h}$ is a 1-cocycle which is called a 1-coboundary; denoting $B^{1}(\mathfrak{k}, \pi)$ the set of 1-coboundaries, put

$$
H^{1}(\mathfrak{k}, \pi)=Z^{1}(\mathfrak{k}, \pi) / B^{1}(\mathfrak{k}, \pi) .
$$

which denotes the first cohomology group of $(\mathfrak{k}, \pi)$.

For $\alpha \in C^{2}(\mathfrak{k}, \pi)$ let

$$
d \alpha(x, y, z)=\sum_{c} \alpha([x, y], z)-\sum_{c} \pi(x) \alpha(y, z) \quad x, y, z \in \mathfrak{k}
$$

where $\sum_{c}$ denotes summation over the set of cyclic permutations of $x, y, z \in \mathfrak{k}$. It is easy to verify that $d \alpha \in C^{3}(\mathfrak{k}, \pi)$, the map $d: C^{2}(\mathfrak{k}, \pi) \rightarrow C^{3}(\mathfrak{k}, \pi)$ is linear so that the set of 2 -cocycles is

$$
Z^{2}(\mathfrak{k}, \pi)=\left\{\alpha \in C^{2}(\mathfrak{k}, \pi): d \alpha=0\right\}
$$

which is the kernel of $d$. On the other hand if $\theta \in C^{1}(\mathfrak{k}, \pi)$ then $d^{2} \theta=0$.

Thus $d$ maps $C^{1}(\mathfrak{k}, \pi)$ onto a subspace of $C^{2}(\mathfrak{k}, \pi)$, denoted by $B^{2}(\mathfrak{k}, \pi)$ and called the 2-coboundaries. Let

$$
H^{2}(\mathfrak{k}, \pi)=Z^{2}(\mathfrak{k}, \pi) / B^{2}(\mathfrak{k}, \pi),
$$

be the second cohomology group.

Remark. One extends the operator $d: C^{s}(\mathfrak{k}, \pi) \rightarrow C^{s+1}(\mathfrak{k}, \pi)$ and one defines the scohomology group as

$$
H^{s}(\mathfrak{k}, \pi)=Z^{s}(\mathfrak{k}, \pi) / B^{s}(\mathfrak{k}, \pi),
$$

where $Z^{s}(\mathfrak{k}, \pi)$ denotes the kernel of $d: C^{s}(\mathfrak{k}, \pi) \rightarrow C^{s+1}(\mathfrak{k}, \pi)$ and $B^{s}(\mathfrak{k}, \pi)=d\left(C^{s-1}(\mathfrak{k}, \pi)\right)$ denotes the image of $d: C^{s-1}(\mathfrak{k}, \pi) \rightarrow C^{s}(\mathfrak{k}, \pi)$, whose elements are called $s$-coboundaries.

Example 2.1. Let $\mathfrak{g}$ be a fixed Lie algebra, and let ad denote the adjoint representation of $\mathfrak{g}$, where $\operatorname{ad}(x) y=[x, y]$ for all $x, y \in \mathfrak{g}$. The Jacobi identity says that the adjoint action acts by derivations of $\mathfrak{g}$. In the setting above, take $\mathfrak{h}=\mathfrak{k}=\mathfrak{g}$, so that $C^{1}(\mathfrak{g}$, ad) denotes the set of linear morphisms $t: \mathfrak{g} \rightarrow \mathfrak{g}$ and for $s \geq 2$, let $C^{s}(\mathfrak{g}$, ad) $=\{\alpha$ : $\alpha$ is s-linear and alternanting on $\mathfrak{g}\}$. For $s \geq 1$ the coboundary operator $d: C^{s}(\mathfrak{g}$, ad $) \rightarrow$ $C^{s+1}(\mathfrak{g}$, ad) (see (1) for $s=1$ and (3) for $s=2$ ) induces the Chevalley cohomology.

Let $\left(\mathfrak{h},[\cdot, \cdot]_{\mathfrak{h}}\right)$ and $\left(\mathfrak{k},[\cdot, \cdot]_{\mathfrak{k}}\right)$ denote real Lie algebras, and let $\pi: \mathfrak{k} \rightarrow \operatorname{End}(\mathfrak{h})$ be a representation. Let $\mathfrak{g}$ be the vector space direct sum of $\mathfrak{h}$ and $\mathfrak{k}$. We would like to define a Lie algebra structure on $\mathfrak{g}=\mathfrak{k} \oplus \mathfrak{h}$ for which $\mathfrak{h}$ is an ideal. 
Define a skew symmetric bilinear map $[\cdot, \cdot]: \mathfrak{g} \times \mathfrak{g} \rightarrow \mathfrak{g}$ by

$$
\begin{aligned}
{[x, y] } & =[x, y]_{\mathfrak{h}} & & x, y \in \mathfrak{h}, \\
{[x, y] } & =\pi(x) y & & x \in \mathfrak{k}, y \in \mathfrak{h}, \\
{[x, y] } & =[x, y]_{\mathfrak{k}}+\alpha(x, y) & & x, y \in \mathfrak{k},
\end{aligned}
$$

where $\alpha: \mathfrak{k} \times \mathfrak{k} \rightarrow \mathfrak{h}$ is bilinear skew-symmetric.

This is a Lie bracket on $\mathfrak{g}$ if and only if the Jacobi identity holds:

$$
[[x, y], z]+[[y, z], x]+[[z, x], y]=0 \quad \text { for all } x, y, z \in \mathfrak{g} .
$$

Thus

- for $x, y, z \in \mathfrak{h},(5)$ follows from the Jacobi identity for $[\cdot, \cdot]_{\mathfrak{h}}$;

- for $x, y \in \mathfrak{h}, z \in \mathfrak{k},(5)$ is satisfied if and only if $\pi(z)$ is a derivation for every $z \in \mathfrak{k}$ :

$$
\pi(z)[x, y]_{\mathfrak{h}}=[\pi(z) x, y]_{\mathfrak{h}}+[x, \pi(z) y]_{\mathfrak{h}} ;
$$

- for $x, y \in \mathfrak{k}, z \in \mathfrak{h}$, since $\pi$ is a representation, the Jacobi identity reduces to

$$
[\alpha(x, y), z]_{\mathfrak{h}}=0,
$$

whence (5) is satisfied in this case if and only if $\alpha$ takes values in the center of $\mathfrak{h}$, denoted by $\mathfrak{z}(\mathfrak{h})$.

- for $x, y, z \in \mathfrak{k}$ we have

$$
[[x, y], z]=\left[[x, y]_{\mathfrak{k}}+\alpha(x, y), z\right]=\left[[x, y]_{\mathfrak{k}}, z\right]_{\mathfrak{k}}+\alpha\left([x, y]_{\mathfrak{k}}, z\right)-\pi(z) \alpha(x, y)
$$

therefore (5) holds if and only if

$$
\begin{aligned}
0= & {\left[[x, y]_{\mathfrak{k}}, z\right]_{\mathfrak{k}}+\left[[y, z]_{\mathfrak{k}}, x\right]_{\mathfrak{k}}+\left[[z, x]_{\mathfrak{k}}, y\right]_{\mathfrak{k}} } \\
0= & \alpha\left([x, y]_{\mathfrak{k}}, z\right)-\pi(z) \alpha(x, y)-\pi(x) \alpha(y, z)+ \\
& +\alpha\left([y, z]_{\mathfrak{k}}, x\right)+\alpha\left([z, x]_{\mathfrak{k}}, y\right)-\pi(y) \alpha(z, x),
\end{aligned}
$$

since $[\cdot, \cdot]_{\mathfrak{k}}$ is a Lie bracket on $\mathfrak{k}$, the first equality is true. For the second one we ask $\alpha$ to be a 2-cocycle from $(\mathfrak{k}, \pi)$.

The above paragraph proves the following proposition.

Proposition 2.2. Let $\left(\mathfrak{h},[\cdot, \cdot]_{\mathfrak{h}}\right)$ and $\left(\mathfrak{k},[\cdot, \cdot]_{\mathfrak{k}}\right)$ be Lie algebras. Let $\pi$ denote a representation from $\mathfrak{k}$ into $\mathfrak{h}$ acting by derivations and let $\alpha \in Z^{2}(\mathfrak{k}, \pi)$. For $\mathfrak{g}=\mathfrak{k} \oplus \mathfrak{h}$ direct sum as vector spaces, the bracket $[\cdot, \cdot]: \mathfrak{g} \times \mathfrak{g} \rightarrow \mathfrak{g}$ as in (4) satisfies the Jacobi identity if and only if

- the image of $\alpha$ is in the center of $\mathfrak{h}: \operatorname{Im} \alpha \subset \mathfrak{z}(\mathfrak{h})$, and

- $\alpha \in Z^{2}(\mathfrak{k}, \pi)$ :

$$
0=\alpha\left([x, y]_{\mathfrak{k}}, z\right)+\alpha\left([y, z]_{\mathfrak{k}}, x\right)+\alpha\left([z, x]_{\mathfrak{k}}, y\right)-\pi(z) \alpha(x, y)-\pi(x) \alpha(y, z)-\pi(y) \alpha(z, x) .
$$

We call the resulting Lie algebra $\mathfrak{g}=\mathfrak{h} \oplus \mathfrak{k}$ as the extended semidirect product of $\mathfrak{h}$ and $\mathfrak{k}$ via $(\pi, \alpha)$. Thus one gets the short exact sequence:

$$
0 \longrightarrow \mathfrak{h} \longrightarrow \mathfrak{g} \longrightarrow \mathfrak{g} / \mathfrak{h} \longrightarrow 0 .
$$

Examples 2.3. In the setting above

- for $\alpha=0$ one gets the semidirect product of $\mathfrak{h}$ and $\mathfrak{k}$ via $\pi$. We shall denote the semidirect product as $\mathfrak{g}=\mathfrak{k} \ltimes_{\pi} \mathfrak{h}$.

In particular the tangent Lie algebra is $T \mathfrak{k}=\mathfrak{k} \ltimes_{\text {ad }} \mathfrak{h}$ where $\mathfrak{h}$ is the underlying vector space to $\mathfrak{k}$ and $\pi=$ ad the adjoint representation. The cotangent Lie algebra 
$\mathbf{T}^{*} \mathfrak{k}=\mathfrak{k} \ltimes_{\text {ad }^{*}} \mathfrak{h}$ where $\mathfrak{h}$ is the underlying vector space to $\mathfrak{k}$ and $\pi=\operatorname{ad}^{*}$ the coadjoint representation.

- $\mathfrak{h}$ abelian and $\pi=0$, one gets a central extension of $\mathfrak{k}$;

- $\mathfrak{h}$ and $\mathfrak{k}$ abelian and $\pi=0$, then $\mathfrak{g}$ is a 2-step nilpotent Lie algebra.

Proposition 2.4. Let $(\mathfrak{g},[\cdot, \cdot])$ be a Lie algebra which decomposes as a direct sum of the vector spaces $\mathfrak{k}$ and $\mathfrak{h}$ where $\mathfrak{h}$ is an ideal in $\mathfrak{g}$. Then $\mathfrak{k}$ can be endowed with a Lie bracket $[\cdot, \cdot]_{\mathfrak{k}}$ in such way that $\mathfrak{g}=\mathfrak{k} \oplus \mathfrak{h}$ is isomorphic to an extended semidirect product as constructed above for a suitable pair $(\pi, \alpha)$.

Proof. Let $[\cdot, \cdot]_{\mathfrak{h}}:=[\cdot, \cdot]_{\mathfrak{h} \times \mathfrak{h}}$ be the restriction of the Lie bracket of $\mathfrak{g}$ to $\mathfrak{h}$. The Jacobi identity in $\mathfrak{g}$ implies that $[\cdot, \cdot]_{\mathfrak{h}}$ is a Lie bracket.

Let $\mathfrak{g}=\mathfrak{k} \oplus \mathfrak{h}$ be a direct sum as vector spaces and let $p_{1}: \mathfrak{g} \rightarrow \mathfrak{k}$ and $p_{2}: \mathfrak{g} \rightarrow \mathfrak{h}$ denote the linear projections with respect to the splitting $\mathfrak{g}=\mathfrak{k} \oplus \mathfrak{h}$.

Since $\mathfrak{h}$ is an ideal in $\mathfrak{g}$, the quotient space $\mathfrak{g} / \mathfrak{h}$ has a Lie algebra structure with Lie bracket $[\cdot, \cdot]^{\prime}$ for which the projection $p: \mathfrak{g} \rightarrow \mathfrak{g} / \mathfrak{h}$ is a homomorphism of Lie algebras: $[p x, p y]^{\prime}=p[x, y]$ for all $x, y \in \mathfrak{g}$. Furthermore the restriction of $p$ to $\mathfrak{k}, p: \mathfrak{k} \rightarrow \mathfrak{g} / \mathfrak{h}$ is a linear isomorphism and for $x, y \in \mathfrak{k}$ one has $p[x, y]=p p_{1}[x, y]$ (identifying $\mathfrak{k}$ with the set $\{(x, 0) \in \mathfrak{g}: x \in \mathfrak{k}\}$.

Let $[\cdot, \cdot]_{\mathfrak{k}}$ be the skew symmetric bilinear form on $\mathfrak{k}$ given by $[x, y]_{\mathfrak{k}}=p^{-1}[p x, p y]^{\prime}$; it satisfies the Jacobi identity since it is the translation of the Lie bracket $[\cdot, \cdot]^{\prime}$ by way of $p$. Thus $\left(\mathfrak{k},[\cdot, \cdot]_{\mathfrak{k}}\right)$ and $\left(\mathfrak{g} / \mathfrak{h},[\cdot, \cdot]^{\prime}\right)$ are isomorphic as Lie algebras. Let $\alpha: \mathfrak{k} \times \mathfrak{k} \rightarrow \mathfrak{h}$ defined by $\alpha(x, y):=p_{2}([x, y])$, this is clearly bilinear and skew symmetric. Moreover (via identifications) the relation

$$
[x, y]=[x, y]_{\mathfrak{k}}+\alpha(x, y)
$$

holds. Finally for $x \in \mathfrak{k}, y \in \mathfrak{h}$ define $\pi(x) y:=[x, y]$.

For $x, y \in \mathfrak{k}, z \in \mathfrak{h}$, the Jacobi identity in $\mathfrak{g}$ implies that $\pi: \mathfrak{k} \rightarrow \operatorname{End}(\mathfrak{h})$ is a homomorphism of Lie algebras and $\operatorname{Im} \alpha \subset \mathfrak{z}(\mathfrak{h})$.

For $x, y \in \mathfrak{h}, z \in \mathfrak{k}$ one has $[z,[x, y]]=\pi(z)[x, y]_{\mathfrak{h}}$, therefore the Jacobi identity for $[\cdot, \cdot]$ says that $\pi$ acts by derivations.

For $x, y, z \in \mathfrak{k}$ we have $[[x, y], z]=\left[[x, y]_{\mathfrak{k}}, z\right]_{\mathfrak{k}}+\alpha\left([x, y]_{\mathfrak{k}}, z\right)-\pi(z) \alpha(x, y)$. Hence the Jacobi identity on $\mathfrak{g}$ implies that $\alpha$ is a 2-cocycle $\alpha \in Z^{2}(\mathfrak{k}, \pi)$. Finally let $\tilde{\mathfrak{g}}$ denote the Lie algebra constructed from $\left(\mathfrak{k},[\cdot, \cdot]_{\mathfrak{k}}\right)$ and $\left(\mathfrak{h},[\cdot, \cdot]_{\mathfrak{h}}\right)$ attached to $(\pi, \alpha)$ then $i_{1}+i_{2}: \tilde{\mathfrak{g}} \rightarrow \mathfrak{g}$, given by $\left(i_{1}+i_{2}\right)(x, y)=x+y \in \mathfrak{g}$ is an isomorphism of Lie algebras, in fact:

$$
\begin{aligned}
\left(i_{1}+i_{2}\right)\left[\left(x_{1}, y_{1}\right),\left(x_{2}, y_{2}\right)\right]= & \left(i_{1}+i_{2}\right)\left(\left[x_{1}, x_{2}\right]_{\mathfrak{k}},\left[y_{1}, y_{2}\right]_{\mathfrak{h}}+\alpha\left(x_{1}, x_{2}\right)+\right. \\
& \left.+\pi\left(x_{1}\right) y_{2}-\pi\left(x_{2}\right) y_{1}\right) \\
= & {\left[x_{1}, x_{2}\right]_{\mathfrak{k}}+\left[y_{1}, y_{2}\right]_{\mathfrak{h}}+\alpha\left(x_{1}, x_{2}\right)+\pi\left(x_{1}\right) y_{2}-\pi\left(x_{2}\right) y_{1} } \\
= & {\left[x_{1}+y_{1}, x_{2}+y_{2}\right] . }
\end{aligned}
$$

Remark. It turns out that the structure above describes an almost product structure on $\mathfrak{g}$ : a linear endomorphism $E: \mathfrak{g} \rightarrow \mathfrak{g}$ satisfying $E^{2}=1$ (and $E \neq 1$ ). Indeed $E$ can be described in terms of its eigenspaces: $\mathfrak{g}=\mathfrak{g}_{+} \oplus \mathfrak{g}_{-}$where $E_{\mid \mathfrak{g}_{+}}=1$ and $E_{\mid \mathfrak{g}_{-}}=-1$.

When $\operatorname{dim} \mathfrak{g}_{+}=\operatorname{dim} \mathfrak{g}_{-}$the almost product structure $E$ is called an almost paracomplex structure. 
The almost product structure $E$ is integrable if

$$
[E x, E y]=-[x, y]+E[E x, y]+E[x, E y] \quad \text { for all } x, y \in \mathfrak{g}
$$

or equivalently the subspaces $\mathfrak{g}_{+}, \mathfrak{g}_{-}$are subalgebras. In this situation $E$ is called a product structure or a paracomplex structure. See for instance [5, 15] and references therein.

\section{Complex structures on Lie Algebras And ideals}

In this section we study the extension problem of complex structures attached to ideals. We consider two situations: the ideal is either invariant by the complex structure or it is totally real.

An almost complex structure on the Lie algebra $\mathfrak{g}$ is an element $J \in V^{1}(\mathfrak{g}$, ad) satisfying $J^{2}=-1$ (where 1 is the identity map). The Nijenhuis tensor for $J$ is defined as

$$
N_{J}(x, y)=[J x, J y]-J d J(x, y) \quad x, y \in \mathfrak{g}
$$

Any almost complex structure $J$ is called integrable if $N_{J} \equiv 0$, that is

$$
d(J)(x, y)=J^{-1}[J x, J y] \quad \text { for all } x, y \in \mathfrak{g},
$$

or explicitly $[J x, J y]=[x, y]+J[J x, y]+J[x, J y]$.

The Nijenhuis tensor verifies, for all $x, y \in \mathfrak{g}$ the following identities:

$$
N_{J}(y, x)=-N_{J}(x, y)=-N_{J}(J x, J y) \quad N_{J}(J x, y)=N_{J}(x, J y)=-J N_{J}(x, y) .
$$

Hence if $\mathfrak{g}$ decomposes into a direct sum of vector subspaces $\mathfrak{g}=\mathfrak{u} \oplus J \mathfrak{u}$, then $N_{J} \equiv 0$ if and only if $N_{J}(x, y)=0$ for all $x, y \in \mathfrak{u}$. As usual, integrable almost complex structures are called complex structures.

Almost complex structures $J: \mathfrak{g} \rightarrow \mathfrak{g}$ satisfying one of the following conditions for any $x, y \in \mathfrak{g}:$

$$
\begin{aligned}
& \text { c1) } J[x, y]=[x, J y] \\
& \text { c2) }[J x, J y]=[x, y]
\end{aligned}
$$

are always integrable. Complex structures of type c1) determine a structure of complex Lie algebra on $\mathfrak{g}$, they are sometimes called bi-invariant. Structures of type c2) are called abelian.

One has the following equivalence relation between Lie algebras with complex structures. Lie algebras with complex structures $\left(\mathfrak{g}_{1}, J_{1}\right)$ and $\left(\mathfrak{g}_{2}, J_{2}\right)$ are called equivalent if there exists an isomorphism of Lie algebras $\sigma: \mathfrak{g}_{1} \rightarrow \mathfrak{g}_{2}$ such that $J_{2} \circ \sigma=\sigma \circ J_{1}$. In particular when $\mathfrak{g}_{1}=\mathfrak{g}_{2}$ a classification of complex structures can be done. See [32, 29] for the classification in dimension four.

If $J^{\prime}=\sigma J \sigma^{-1}$, by using that $\sigma$ is an automorphism one gets that $J^{\prime}$ is abelian (biinvariant) if $J$ is of this type.

Let $\mathfrak{v} \subseteq \mathfrak{g}$ be a subspace on a Lie algebra $\mathfrak{g}$ equipped with a complex structure $J$, recall that $\mathfrak{v}$ is called

$$
\begin{array}{ll}
\text { complex if } & J \mathfrak{v} \subseteq \mathfrak{v}, \\
\text { totally real if } & \mathfrak{v} \cap J \mathfrak{v}=\{0\} .
\end{array}
$$

Now we are interested in studying complex structures on extended semidirect products $\mathfrak{g}=\mathfrak{k} \oplus \mathfrak{h}$ attached to $(\pi, \alpha)$, specifically when the ideal $\mathfrak{h}$ is either complex or totally real. 
A complex ideal. Let $\mathfrak{g}$ be a Lie algebra such that $\mathfrak{h} \subset \mathfrak{g}$ is an ideal. Let $\mathfrak{k} \subset \mathfrak{g}$ be a complementary subspace of $\mathfrak{g}$ attached with the pair $(\pi, \alpha)$ and endowed with the algebraic structure given in Proposition 2.4.

Assume there is a complex structure $J$ on $\mathfrak{g}$ such that $\mathfrak{h}$ is $J$-invariant. In terms of the direct sum as vector spaces $\mathfrak{g}=\mathfrak{k} \oplus \mathfrak{h}$, we notice that the subspace $\mathfrak{k}$ is not necessarily $J$-invariant. Thus for $x \in \mathfrak{k}$ one has:

$$
J(x)=j(x)+\beta(x) \quad \text { where } j: \mathfrak{k} \rightarrow \mathfrak{k} \text { and } \beta: \mathfrak{k} \rightarrow \mathfrak{h} \text { is linear. }
$$

Since $J^{2}=-1$ one gets

$$
J^{2}(x)=-x=j^{2}(x)+\beta(j(x))+J \beta(x)
$$

and this implies

$$
j: \mathfrak{k} \rightarrow \mathfrak{k} \text { is an almost complex structure and }
$$

$$
J \beta(x)=-\beta(j(x)) \quad \forall x \in \mathfrak{k} .
$$

Now the integrability condition of $J$ says:

- For $x, y \in \mathfrak{h}$

$$
[J x, J y]_{\mathfrak{h}}=[x, y]_{\mathfrak{h}}+J[J x, y]_{\mathfrak{h}}+J[x, J y]_{\mathfrak{h}}
$$

which is the integrability condition for the restriction $J_{\left.\right|_{\mathfrak{h}}}: \mathfrak{h} \rightarrow \mathfrak{h}$.

- For $x \in \mathfrak{k}, y \in \mathfrak{h}$, on the one hand

$$
[J x, J y]=[j(x)+\beta(x), J y]=\pi(j(x)) J y+[\beta(x), J y]_{\mathfrak{h}}
$$

and on the other hand

$$
\begin{aligned}
{[x, y]+J[J x, y]+J[x, J y] } & =\pi(x) y+J[j(x)+\beta(x), y]+J[x, J y] \\
& =\pi(x) y+J \pi(j(x)) y+J[\beta(x), y]_{\mathfrak{h}}+J \pi(x) J y
\end{aligned}
$$

hence

$$
\pi(j(x)) J y+[\beta(x), J y]_{\mathfrak{h}}=\pi(x) y+J \pi(j(x)) y+J \pi(x) J y+J[\beta(x), y]_{\mathfrak{h}} .
$$

- For $x, y \in \mathfrak{k}$ : on the one hand

$$
\begin{aligned}
{[J x, J y] } & =[j(x)+\beta(x), j y+\beta(y)] \\
& =[j(x), j(y)]_{\mathfrak{k}}+\alpha(j(x), j(y))+\pi(j(x)) \beta(y)-\pi(j(y)) \beta(x)+[\beta(x), \beta(y)]_{\mathfrak{h}}
\end{aligned}
$$

while on the other side

$$
\begin{aligned}
{[J x, J y]=} & {[x, y]_{\mathfrak{k}}+\alpha(x, y)+J[j(x)+\beta(x), y]+J[x, j(y)+\beta(y)] } \\
= & {[x, y]_{\mathfrak{k}}+\alpha(x, y)+J\left([j(x), y]_{\mathfrak{k}}-\alpha(j(x), y)-\pi(y) \beta(x)\right) } \\
& +J\left([x, j(y)]_{\mathfrak{k}}+\alpha(x, j(y))+\pi(x) \beta(y)\right) \\
= & {\left.[x, y]_{\mathfrak{k}}+\alpha(x, y)+j[j(x), y]_{\mathfrak{k}}+\beta\left([j(x), y]_{\mathfrak{k}}\right)+J \alpha(j(x), y)-J \pi(y) \beta(x)\right) } \\
& \left.+j[x, j(y)]_{\mathfrak{k}}+\beta\left([x, j y]_{\mathfrak{k}}\right)+J \alpha(x, j(y))+J \pi(x) \beta(y)\right) .
\end{aligned}
$$

Comparing both expressions one can see that the $\mathfrak{k}$-component of the equality above must satisfy

$$
[j(x), j(y)]_{\mathfrak{k}}=[x, y]_{\mathfrak{k}}+j[j(x), y]_{\mathfrak{k}}+j[x, j(y)]_{\mathfrak{k}}
$$

for an almost $j \in \operatorname{End}(\mathfrak{k})$ such that $j^{2}=-1$.

While the $\mathfrak{h}$-component involves all the elements: $[,]_{\mathfrak{h}}, \alpha, \beta, j$ and $J$ :

$$
\alpha(j(x), j(y))-\alpha(x, y)-J \alpha(x, j(y))-J \alpha(j(x), y)=
$$




$$
\begin{aligned}
= & \beta\left([j(x), y]_{\mathfrak{k}}\right)+\beta\left([x, j y]_{\mathfrak{k}}\right)-[\beta(x), \beta(y)]_{\mathfrak{h}}+ \\
& +\pi(j(y)) \beta(x)-\pi(j(x)) \beta(y)-J \pi(y) \beta(x))+J \pi(x) \beta(y) .
\end{aligned}
$$

Conversely one has

Proposition 3.1. Let $(\mathfrak{h}, J)$ denote a Lie algebra equipped with a complex structure $J$. Let $\mathfrak{g}=\mathfrak{k} \oplus \mathfrak{h}$ denote a Lie algebra such that $\mathfrak{h}$ is an ideal of $\mathfrak{g}$ and $\mathfrak{k}$ is a linear subspace and let $(\pi, \alpha)$ be the elements arising from the exact sequence

$$
0 \longrightarrow \mathfrak{h} \longrightarrow \mathfrak{g} \longrightarrow \mathfrak{g} / \mathfrak{h} \longrightarrow 0
$$

as in Proposition 2.4.

Let $j: \mathfrak{k} \rightarrow \mathfrak{k}$ denote an almost complex structure on $\mathfrak{k}$ and define $\tilde{J}: \mathfrak{g} \rightarrow \mathfrak{g}$ by

$$
\begin{aligned}
& \tilde{J} x=J x \quad \text { for } x \in \mathfrak{h} \\
& \tilde{J} x=j x+\beta(x) \quad \text { for } x \in \mathfrak{k}
\end{aligned}
$$

where $\beta \in \operatorname{Hom}(\mathfrak{k}, \mathfrak{h})$. Then $\tilde{J}$ defines a complex structure on $\mathfrak{g}$ if and only if (8)), (9), (10), (11) hold.

Note that for different reasons the subspace $\mathfrak{k}$ above could not be $J$-invariant. For instance in presence of a symplectic structure it could be necessary to take it isotropic but not complex.

Assume now the subspace $\mathfrak{k}$ is $J$-invariant. Then the starting point for the construction is the above one with $\beta=0$. Let $J_{1}: \mathfrak{k} \rightarrow \mathfrak{k}$ an almost complex structure and $J_{2}: \mathfrak{h} \rightarrow \mathfrak{h}$ also an almost complex structure. Let $\mathfrak{g}=\mathfrak{k} \oplus \mathfrak{h}$ as in Proposition 2.2. The linear map $J_{ \pm}=\left(J_{1}, \pm J_{2}\right)$ defines an almost complex structure on $\mathfrak{g}$.

Thus the Nijenhuis tensor on $\mathfrak{k}$ and $\mathfrak{h}$ gives that

- $N_{J_{ \pm}}(x, y)=N_{J_{1}}(x, y)$ for all $x, y \in \mathfrak{k}$;

- $N_{J_{ \pm}}(x, y)=N_{J_{2}}(x, y)$ for all $x, y \in \mathfrak{h}$.

The proof of the following corollary follows from the situation above in the case $\beta=0$.

Corollary 3.2. Let $\mathfrak{g}=\mathfrak{k} \oplus \mathfrak{h}$ be a Lie algebra as in (2.2) attached to $(\pi, \alpha)$ and let $J_{1}$ denote a complex structure on $\mathfrak{k}$ and $J_{2}$ a complex on $\mathfrak{h}$. The almost complex structure on $\mathfrak{g}$ given by

$$
J_{ \pm}(x, y):=\left(J_{1} x, \pm J_{2}\right)
$$

is integrable if and only if the following conditions hold

(i) $\varepsilon\left[\pi\left(J_{1} x\right), J_{2}\right] y+\left[\pi(x), J_{2}\right] J_{2} y=0 \quad$ for $x \in \mathfrak{k}, y \in \mathfrak{h}$;

(ii) $\alpha\left(J_{1} x, J_{1} y\right)-\alpha(x, y)+\varepsilon J_{2}\left(\alpha\left(J_{1} x, y\right)+\alpha\left(x, J_{1} y\right)\right)=0 \quad$ for $x, y \in \mathfrak{k}$, where $\varepsilon=1$ for $J_{+}$and $\varepsilon=-1$ for $J_{-}$.

Definition 3.3. Let $J_{1}$ be a complex structure on $\mathfrak{k}$ and $J_{2}$ be a complex structure on a vector space $V$. Assume $\mathfrak{k}$ acts on $V$ via $\pi$. We shall say that the action is holomorphic if $\left[\pi(x), J_{2}\right]=0$ for all $x \in \mathfrak{k}$.

Let $B: \mathfrak{k} \times \mathfrak{k} \rightarrow V$ be a bilinear map. We say that $B$ is compatible with $J_{1}$ if $B\left(J_{1} x, J_{1} y\right)=$ $B(x, y)$.

Corollary 3.4. Let $\mathfrak{g}=\mathfrak{k} \oplus \mathfrak{h}$ be a Lie algebra as in (2.2) attached to $(\pi, \alpha)$. Let $J_{1}$ denote a complex structure on $\mathfrak{k}$ and $J_{2}$ a complex structure on $\mathfrak{h}$. Assume that the action of $\mathfrak{k}$ into $\mathfrak{h}$ is holomorphic and $\alpha$ is compatible with $J_{1}$. Then the almost complex structure $J_{ \pm}(x, y):=\left(J_{1} x, \pm J_{2} y\right)$ is integrable on $\mathfrak{g}$. 
At the Lie group level one has the next result. See [31] for more details.

Lemma 3.5. 31] Let $(\mathfrak{g}, J)$ be a Lie-algebra with complex structure. Let $\mathfrak{h} \subset \mathfrak{g}$ be an ideal of $\mathfrak{g}$ such is complex. Let $G$ and $H$ denote the associated simply connected Lie-groups endowed with the left-invariant complex structures induced by $J$ and assume thta $H$ is closed in $G$. Then there is a holomorphic fibration $\rho: G \rightarrow G / H$ with fiber $H$.

A totally real ideal. Now we study complex structures $J$ on a Lie algebra of the form $\mathfrak{g}=\mathfrak{k} \oplus \mathfrak{h}$ where $\mathfrak{h}$ is an ideal in $\mathfrak{g}$ and such that $J \mathfrak{k}=\mathfrak{h}$.

Examples of this can be constructed from 1-cocycles as we show below. Let $\mathfrak{k}$ be a Lie algebra and let $\mathfrak{h}$ be a 2 -step nilpotent Lie algebra of the same dimension as $\mathfrak{k}$. Let $\pi$ be a representation of $\mathfrak{k}$ into $\mathfrak{h}$ by derivations and let $j \in Z^{1}(\mathfrak{k}, \pi)$ be of maximal rank.

Let $\alpha_{j}: \mathfrak{k} \times \mathfrak{k} \rightarrow \mathfrak{z}(\mathfrak{h})$ be the skew symmetric bilinear map given by

$$
\alpha_{j}(x, y)=[j x, j y]_{\mathfrak{h}}
$$

This is a 2-cocycle, in fact using that $j$ is a 1-cocycle and $\pi$ acts by derivations, for $x, y, z \in \mathfrak{k}$ one has

$$
\begin{aligned}
d \alpha_{j}(x, y, z)= & {\left[j[x, y]_{\mathfrak{k}}, j z\right]_{\mathfrak{h}}+\left[j[y, z]_{\mathfrak{k}}, j x\right]_{\mathfrak{h}}+\left[j[z, x]_{\mathfrak{k}}, j y\right]_{\mathfrak{h}}+} \\
& -\pi(x)[j y, j z]_{\mathfrak{h}}-\pi(y)[j z, j x]_{\mathfrak{h}}-\pi(z)[j x, j y]_{\mathfrak{h}} \\
= & {[\pi(x) j y-\pi(y) j x, j z]_{\mathfrak{h}}+[\pi(y) j z-\pi(z) j y, j x]_{\mathfrak{h}}+} \\
& {[j y, \pi(z) j x-\pi(x) j z]_{\mathfrak{h}}-[\pi(x) j y, j z]_{\mathfrak{h}}-[j y, \pi(x) j z]_{\mathfrak{h}}+} \\
& -[\pi(y) j z, j x]_{\mathfrak{h}}-[j z, \pi(y) j x]_{\mathfrak{h}}-[\pi(z) j x, j y]_{\mathfrak{h}}-[j x, \pi(z) j y]_{\mathfrak{h}} \\
= & 0 .
\end{aligned}
$$

Thus Proposition 2.2 says that $\mathfrak{g}=\mathfrak{k} \oplus \mathfrak{h}$ attached to $\left(\pi, \alpha_{j}\right)$ is a Lie algebra with Lie bracket $[\cdot, \cdot]$ as in (41). Moreover the almost complex structure $J: \mathfrak{g} \rightarrow \mathfrak{g}$ given by

$$
J_{\left.\right|_{\mathfrak{k}}}=j \quad J_{\left.\right|_{\mathfrak{h}}}=-j^{-1}
$$

is integrable. In fact, by calculating the Nijenhuis tensor $N_{J}(x, y)$ for $x, y \in \mathfrak{k}$ one gets

$$
\begin{aligned}
N_{J}(x, y) & =[j x, j y]_{\mathfrak{h}}-[x, y]_{\mathfrak{k}}-\alpha_{j}(x, y)-j \pi(x) j y-j \pi(y) j x \\
& =[j x, j y]_{\mathfrak{h}}-[x, y]_{\mathfrak{k}}-[j x, j y]_{\mathfrak{h}}-j \pi(x) j y-j \pi(y) j x \\
& =0
\end{aligned}
$$

where the last equality follows from the condition of $j$ being a 1-cocycle. These considerations prove the following result.

Proposition 3.6. Let $\mathfrak{g}=\mathfrak{k} \oplus \mathfrak{h}$ be a Lie algebra attached to $(\pi, \alpha)$ as in Proposition [2.2 where $\mathfrak{h}$ is two-step nilpotent and $\operatorname{dim} \mathfrak{h}=\operatorname{dim} \mathfrak{k}$. Let $j \in C^{1}(\mathfrak{k}, \pi)$. Then the endomorphism $J: \mathfrak{g} \rightarrow \mathfrak{g}$, given by

$$
J(x, y)=\left(-j^{-1} y, j x\right) \quad x, y \in \mathfrak{k}
$$

defines a complex structure on $\mathfrak{g}$ if and only if $j$ is a 1-cocycle of maximal rank and the 2-cocycle $\alpha$ satisfies $\alpha(x, y)=[j x, j y]_{\mathfrak{h}}$ for all $x, y \in \mathfrak{k}$.

The converse of the previous construction is given in the following theorem.

Theorem 3.7. Let $\mathfrak{g}$ be a Lie algebra with a complex structure $J$ and assume that $\mathfrak{g}$ decomposes into a direct sum of vector spaces $\mathfrak{g}=\mathfrak{k} \oplus J \mathfrak{k}$ where $J \mathfrak{k}$ is an ideal in $\mathfrak{g}$. Then $\mathfrak{h}:=J \mathfrak{k}$ is either 2-step nilpotent or abelian and $J$ is induced from a 1-cocycle $j \in Z^{1}(\mathfrak{k}, \pi)$ 
of maximal rank, if $\mathfrak{k}$ is equipped with the Lie bracket of $\mathfrak{g} / \mathfrak{h}$ and $\pi$ is a representation from $\mathfrak{k}$ into $\mathfrak{h}$ by derivations.

Proof. Let $\mathfrak{h}:=J \mathfrak{k}$ denote the ideal in $\mathfrak{g}$. According to Proposition 2.4 The Lie algebra $\mathfrak{g}$ is isomorphic to the Lie algebra $\mathfrak{k} \oplus \mathfrak{h}$ attached to $(\pi, \alpha)$, where $\pi(x) y=[x, y]$, for $x \in \mathfrak{k}$, $y \in \mathfrak{h}$ and $\alpha(x, y)=p_{2}[x, y]$ for $x, y \in \mathfrak{k}$, the linear map $p_{2}: \mathfrak{g} \rightarrow \mathfrak{h}$ is the projection onto $\mathfrak{h}$ with respect to the decomposition $\mathfrak{k} \oplus \mathfrak{h}$.

If $J$ is a complex structure on $\mathfrak{g}$, by restricting it to $\mathfrak{k}$, the map $j:=J_{\mid \mathfrak{k}}$ naturally induces an element $j \in C^{1}(\mathfrak{k}, \pi)$ of maximal rank, since $\mathfrak{g}$ decomposes as a direct sum $\mathfrak{g}=\mathfrak{k} \oplus J \mathfrak{k}$. Furthermore, since $J^{2}=-1$, for any $x \in \mathfrak{k}$ we have $-x=J^{2} x=-j^{-1} j x$. Thus one can write $J$ in the form (14).

By computing the Nijenhuis tensor for $x, y \in \mathfrak{k}$ one gets that $j$ is a 1-cocycle and $\alpha(x, y)=[j x, j y]_{\mathfrak{h}}$ for $x, y \in \mathfrak{k}$. Since $\operatorname{Im} \alpha \subset \mathfrak{z}(\mathfrak{h})$, one obtains that the commutator of $\mathfrak{h}$ is contained in the center $C^{1}(\mathfrak{h}) \subset \mathfrak{z}(\mathfrak{h})$, and this says $\mathfrak{h}$ is either two-step nilpotent or abelian if $\alpha \equiv 0$.

Remark. The results here generalize those in [18. In fact the results proved there was the following for tangent Lie algebras. Let $\mathrm{T} \mathfrak{k}$ denote the tangent Lie algebra of a Lie algebra $\mathfrak{k}$. In [18] a complex structure $J$ on $\mathrm{Tk}^{\mathfrak{k}}$ such that $J \mathfrak{k}=\mathfrak{h}$ is called a totally real complex structure.

Theorem 3.8. 18] Let $\mathrm{T}_{\mathfrak{k}}$ denote the tangent Lie algebra of a Lie algebra $\mathfrak{k}$. The set of totally real complex structures on $\mathrm{T}_{\mathfrak{k}}$ is in one to one correspondence with the set of non-singular derivations of $\mathfrak{k}$.

If one set of those (and therefore both) is non-empty then $\mathfrak{k}$ is nilpotent.

Remark. Totally real complex structures on semidirect products of the form $V \rtimes_{\pi} \mathfrak{k}$, where $V$ is the underlying vector space to $\mathfrak{k}$ equipped with its canonical abelian bracket, are in correspondence with Lagrangian symplectic structures on $V \rtimes_{\pi^{*}} \mathfrak{k}$. See e.g. [16].

Example 3.9. In the paragraphs we get examples of an (almost) complex product structure on a Lie algebra $\mathfrak{g}$ : that is a pair $(J, E)$ of an (almost) complex structure $J$ and an (almost) product structure $E$ (Remark 2.1) such that $J E=-E J$. See [8, 11].

\section{Bilinear forms, ideals and complex structures}

In this section we shall study compatibility conditions for $J$ with respect to nondegenerate either symmetric or skew-symmetric bilinear forms on a Lie algebra $\mathfrak{g}$ having a fixed ideal.

4.1. Symmetric case. A metric on a real vector space $\mathfrak{v}$ is a symmetric bilinear map on $\mathfrak{v},\langle\rangle:, \mathfrak{v} \times \mathfrak{v} \rightarrow \mathbb{R}$ which is non-degenerate, that is, for any non zero vector $x \in \mathfrak{v}$ there exists a vector $y \in \mathfrak{v}$ such that $\langle x, y\rangle \neq 0$. Otherwise $\langle$,$\rangle is said degenerate.$

If $\mathfrak{w}$ is a subspace of $(\mathfrak{v},\langle\rangle$,$) the subspace$

$$
\mathfrak{w}^{\perp}=\{x \in \mathfrak{v}:\langle x, v\rangle=0 \quad \text { for all } \quad v \in \mathfrak{w}\}
$$

denotes the orthogonal subspace of $\mathfrak{w}$. In particular we say that $\mathfrak{w}$ is

- isotropic if $\mathfrak{w} \subset \mathfrak{w}^{\perp}$,

- totally isotropic if $\mathfrak{w}=\mathfrak{w}^{\perp}$ and 
- non-degenerate if $\mathfrak{w} \cap \mathfrak{w}^{\perp}=0$.

In the last case, the restricted metric $\langle,\rangle_{\mathfrak{w}}:=\langle,\rangle_{\left.\right|_{\mathfrak{w} \times \mathfrak{w}}}$, defines a isomorphism $\xi$ between $\mathfrak{w}$ and its dual space $\mathfrak{w}^{*}$ by $\xi(u)(v)=\langle u, v\rangle_{\mathfrak{w}}$ whenever $\mathfrak{w}$ is finite dimensional. As usual, a metric of index 0 or signature $(0, n)$ on a vector space of dimension $n$ is called an inner product.

Example 4.1. Let $\mathfrak{u}$ denote a vector space whose dual space is denoted by $\mathfrak{u}^{*}$. Let $\mathfrak{u} \oplus \mathfrak{u}^{*}$ be the direct sum as vector spaces of $\mathfrak{u}$ and $\mathfrak{u}^{*}$ and endow this with the hyperbolic metric $\left\langle x_{1}+\phi_{1}, x_{2}+\phi_{2}\right\rangle=\phi_{1}\left(x_{2}\right)+\phi_{2}\left(x_{1}\right)$ where $\phi_{i} \in \mathfrak{u}^{*}, x_{i} \in \mathfrak{u}$, for $\mathrm{i}=1,2$. Clearly $\mathfrak{u}$ and $\mathfrak{u}^{*}$ are complementary totally isotropic subspaces in $\mathfrak{u} \oplus \mathfrak{u}^{*}$.

Definition 4.2. Let $\langle$,$\rangle denote a metric on a Lie algebra \mathfrak{g}$. Let $J$ denote an (almost) complex structure on $\mathfrak{g}$. The pair $(J,\langle\rangle$,$) defines$

- an Hermitian structure on $\mathfrak{g}$ if $\langle J x, J y\rangle=\langle x, y\rangle \forall x, y \in \mathfrak{g}$,

- an (almost) anti-Hermitian structure on $\mathfrak{g}$ if $\langle J x, J y\rangle=-\langle x, y\rangle \forall x, y \in \mathfrak{g}$.

We shall also say that the metric $\langle$,$\rangle is (almost)-Hermitian or (almost) anti-Hermitian.$

We note that here we consider Hermitian structures in relation to possibly definite metrics, although this notion is referred to by other authors as definite metrics. AntiHermitian structures are also called Norden metrics [28] or B-metrics.

In the next paragraphs we discuss different possible constructions.

Case of a complex ideal. Here we shall consider different metric constructions for the complex ideal $\mathfrak{h}$.

Let $\mathfrak{g}$ denote a Lie algebra with a complex structure $J$ and let $\mathfrak{h} \subset \mathfrak{g}$ be a complex ideal on $\mathfrak{g}$. Assume $\langle$,$\rangle is a metric on \mathfrak{g}$ and $\mathfrak{h}$ is non-degenerate relative to the metric. Let $\mathfrak{k}$ be the orthogonal complementary subspace of $\mathfrak{h}$. Then as in 3.1, the complex structure $J$ does not need to leave $\mathfrak{k}$ invariant. The pair $(J,\langle\rangle$,$) is Hermitian if and only if$

$$
\langle J x, J y\rangle=\langle x, y\rangle \quad \forall x, y \in \mathfrak{g}
$$

which implies

(1) $\langle J x, J y\rangle=\langle x, y\rangle \quad \forall x, y \in \mathfrak{h}$

$$
\begin{aligned}
\langle j(x)+\beta(x), j(y)+\beta(y)\rangle & =\langle j(x), j(y)\rangle+\langle\beta(x), \beta(y)\rangle \\
& =\langle x, y\rangle \quad \forall x, y \in \mathfrak{k}
\end{aligned}
$$

which says that $\left(J_{\left.\right|_{\mathfrak{h}}},\langle,\rangle_{\mathfrak{h}}\right)$ defines an (almost) Hermitian structure on $\mathfrak{h}$ and on $\mathfrak{k}$ one should have:

$$
\langle j(x), j(y)\rangle-\langle x, y\rangle+\langle\beta(x), \beta(y)\rangle=0 \quad \text { for all } x, y \in \mathfrak{k} .
$$

Thus in this situation $\mathfrak{h}$ is a complex non-degenerate ideal and $\mathfrak{k}$ is non-degenerate albeit $J$-invariant.

Some cases are the following ones.

(1) Let $\mathfrak{k}$ and $\mathfrak{h}$ denote Lie algebras with corresponding Hermitian structures $\left(J_{1}, B_{1}\right)$ and $\left(J_{2}, B_{2}\right)$. Let $\mathfrak{g}=\mathfrak{k} \oplus \mathfrak{h}$ be a Lie algebra as in Proposition2.2 attached to $(\pi, \alpha)$, then $\left(J_{ \pm}, B_{1}+B_{2}\right)$ defines an almost Hermitian structure on $\mathfrak{g}$. 
(2) Let $\left(\mathfrak{k}, J_{1}\right)$ and $\left(\mathfrak{h}, J_{2}\right)$ denote Lie algebras with corresponding (almost) complex structures. Assume $\operatorname{dim} \mathfrak{h}=\operatorname{dim} \mathfrak{k}$ and let $t: \mathfrak{k} \rightarrow \mathfrak{h}$ be an isomorphism. Let $B$ be a metric on $\mathfrak{k}$ and consider the metric on $\mathfrak{g}$ given by

$$
\left\langle\left(x_{1}, t y_{1}\right),\left(x_{2}, t y_{2}\right)\right\rangle=B\left(x_{1}, y_{2}\right)+B\left(x_{2}, y_{1}\right) .
$$

Then the pair $\left(J:=\left(J_{1}, \pm J_{2}\right),\langle\rangle,\right)$ defines an (almost) Hermitian structure on $\mathfrak{g}$ if and only if $J_{2} t= \pm t J_{1}$.

Notice that in this situation both $\mathfrak{k}$ and $\mathfrak{h}$ are isotropic subspaces and the metric has signature $(n, n)$ where $n=\operatorname{dim} \mathfrak{k}$.

Corollary 4.3. Let $\mathfrak{k}$ denote a Lie algebra with an (almost) Hermitian structure $\left(J_{1}, B\right)$. Let $\mathfrak{g}=\mathfrak{k} \oplus \mathfrak{h}$ be the extended Lie algebra such that $\mathfrak{h}$ is an ideal of $\mathfrak{g}$. Assume $\operatorname{dim} \mathfrak{h}=\operatorname{dim} \mathfrak{k}$ and let $t: \mathfrak{k} \rightarrow \mathfrak{h}$ be a linear isomorphism. Define an almost structure $J_{2}$ on $\mathfrak{h}$ by

$$
J_{2}=t J_{1} t^{-1}
$$

Then the metric on $\mathfrak{g}$ given by $\left\langle\left(x_{1}, t y_{1}\right),\left(x_{2}, t y_{2}\right)\right\rangle=B\left(x_{1}, y_{2}\right)+B\left(x_{2}, y_{1}\right)$ gives rise to an (almost) Hermitian structure for $J=\left(J_{1}, J_{2}\right)$.

Case of a totally real ideal. Here we shall consider different metric constructions for the totally real ideal $\mathfrak{h}$.

Let $\mathfrak{g}$ be a Lie algebra which splits into a direct sum of vector spaces $\mathfrak{g}=\mathfrak{k} \oplus \mathfrak{h}$ and which admit an almost complex structure $J$ such that $J \mathfrak{k}=\mathfrak{h}$ : i.e. there is a linear isomorphism $j: \mathfrak{k} \rightarrow \mathfrak{h}($ see $(2.2))$.

$$
J(x, y)=\left(-j^{-1} y, j x\right) \quad \text { for all } x \in \mathfrak{k}, y \in \mathfrak{h} .
$$

Let $B$ denote a metric on $\mathfrak{k}$. Consider the following metric $\langle$,$\rangle as extension of B$ to $\mathfrak{g}$ :

$$
\left\langle\left(x_{1}, j y_{1}\right),\left(x_{2}, j y_{2}\right)\right\rangle=B\left(x_{1}, x_{2}\right)+B\left(y_{1}, y_{2}\right) \quad \text { for all } x_{i}, y_{i} \in \mathfrak{k}, i=1,2 .
$$

This metric is Hermitian:

$$
\begin{aligned}
\left\langle J\left(x_{1}, j y_{1}\right), J\left(x_{2}, j y_{2}\right)\right\rangle & =\left\langle\left(-y_{1}, j x_{1}\right),\left(-y_{2}, j x_{2}\right)\right\rangle \\
& =B\left(y_{1}, y_{2}\right)+B\left(x_{1}, x_{2}\right)
\end{aligned}
$$

Clearly $\langle$,$\rangle restricts to both \mathfrak{k}$ and $\mathfrak{h}$ as a metric and so that $\mathfrak{k} \perp \mathfrak{h}$. However the geometry that \langle\rangle determines on $\mathfrak{k}$ is different from that one on $(\mathfrak{k}, B)$.

Let us denote by $\nabla$ the Levi Civita connection corresponding to $\langle$,$\rangle . For$ $x, y, z \in \mathfrak{k}$ the following formulas hold

$$
2\left\langle\nabla_{x} y, j z\right\rangle=\langle\alpha(x, y), z\rangle \quad 2\left\langle\nabla_{j x} j y, z\right\rangle=\langle\pi(z) j y, j x\rangle-\langle\pi(z) j x, j y\rangle
$$

showing that $\mathfrak{k}$ and $\mathfrak{h}$ are not necessarily totally geodesic subspaces.

$$
\left\langle\left(x_{1}, j y_{1}\right),\left(x_{2}, j y_{2}\right)\right\rangle=B\left(x_{1}, y_{2}\right)+B\left(x_{2}, y_{1}\right) \quad \text { for all } x_{i}, y_{i} \in \mathfrak{k}, i=1,2 .
$$

This metric is anti-Hermitian. Both spaces $\mathfrak{k}$ and $\mathfrak{h}$ are isotropic.

Proposition 4.4. Let $(\mathfrak{g}, J)$ be a Lie algebra equipped with an almost complex structure $J$ and assume $\mathfrak{g}$ splits into a direct sum of vector spaces $\mathfrak{g}=\mathfrak{k} \oplus \mathfrak{h}$ such that $J \mathfrak{k}=\mathfrak{h}$. Then $\mathfrak{g}$ always admits an Hermitian and an anti-Hermitian metric for $J$. 
Proof. Let $B$ denote an inner product on $\mathfrak{k}$ and denote by $j$ the restriction of $J$ to $\mathfrak{k}, j:=J_{\mid \mathfrak{k}}$. Since $J \mathfrak{k}=\mathfrak{h}$, the almost complex structure $J$ induces a linear morphism $j: \mathfrak{k} \rightarrow \mathfrak{h}$ which is non singular and since $J^{2}=-1$, it is easy to see that $J$ is related to $j$ by the formula $J(x, y)=\left(-j^{-1} y, j x\right)$.

Let $\langle$,$\rangle be the metric on \mathfrak{g}$ given by

$$
\left\langle\left(x_{1}, y_{1}\right),\left(x_{2}, y_{2}\right)\right\rangle=-B\left(x_{1}, j^{-1} y_{2}\right)-B\left(x_{2}, j^{-1} y_{1}\right) .
$$

The map $\langle$,$\rangle is bilinear symmetric and non-degenerate. Moreover it is compatible with$ $J$, as it follows from

$$
\begin{aligned}
\left\langle J\left(x_{1}, y_{1}\right), J\left(x_{2}, y_{2}\right)\right\rangle & =\left\langle\left(-j^{-1} y_{1}, j x_{1}\right),\left(-j^{-1} y_{2}, j x_{2}\right)\right\rangle \\
& =-B\left(-j^{-1} y_{1}, x_{2}\right)-B\left(-j^{-1} y_{2}, x_{1}\right) \\
& =\left\langle\left(x_{1}, y_{1}\right),\left(x_{2}, y_{2}\right)\right\rangle .
\end{aligned}
$$

Note that the subspaces $\mathfrak{k}$ and $\mathfrak{h}$ are isotropic and of maximal dimension hence they are totally isotropic.

For the anti-Hermitian structure the metric on $\mathfrak{g}$ is defined as

$$
\left\langle\left(x_{1}, y_{1}\right),\left(x_{2}, y_{2}\right)\right\rangle=-B\left(x_{1}, j^{-1} y_{2}\right)+B\left(x_{2}, j^{-1} y_{1}\right)
$$

and the proof follows along the same lines of the preceding case.

A remark on SKT structures Let $(M, J, g)$ be a Hermitian manifold. If the torsion 3 -form $c$ of the Bismut connection is $d$-closed, then the Hermitian metric $g$ on a complex manifold $(M, J)$ is called strong Kähler with torsion (shortly SKT), where

$$
c(x, y, z)=g\left(x, T^{B}(y, z)\right)
$$

being $T^{B}$ the torsion of the Bismut connection $\nabla^{B}$ characterised as the unique connection on the Hermitian manifold $(M, J, g)$ such that $\nabla^{B} J=0, \nabla^{B} g=0$.

Let $\mathfrak{s o}(\mathfrak{g}, g)$ denote the Lie algebra of skew symmetric maps for $g$. See 21] for the proof of the next result and for more details and references on SKT structures.

Proposition 4.5. 21] Let $(\mathfrak{g}, J, g)$ be a Hermitian Lie algebra and let $\pi: \mathfrak{g} \rightarrow \mathfrak{s o}(\mathfrak{g}, g)$ be a representation such that $\pi$ is holomorphic. Take $\mathfrak{g} \ltimes_{\pi} \mathfrak{h}$ where $\mathfrak{h}$ is the vector space underlying $\mathfrak{g}$ with the trivial bracket. Then the Hermitian structure $(\tilde{J}, \tilde{g})$ given by $\tilde{g}=g+g$ the product metric on $\mathfrak{g} \oplus \mathfrak{h}$ and $\tilde{J}(x, y)=(J x, J y)$ is SKT if and only if $(J, g)$ is SKT on $\mathfrak{g}$.

In remark 3.2 the authors exemplify the result for the adjoint representation. They say that the conditions of the Proposition hold in this situation if and only if on the Hermitian Lie algebra $(\mathfrak{g}, J, g)$ the complex structure $J$ is bi-invariant and the inner product $g$ is adinvariant. However as proved in [6] this is possible only for an abelian Lie algebra $\mathfrak{g}$.

4.2. Skew-symmetric case. Here we shall study symplectic structures on Lie algebras $\mathfrak{g}$ with an ideal $\mathfrak{h}$.

A symplectic structure on a Lie algebra $\mathfrak{g}$ is a skew-symmetric non-degenerate bilinear form on $\mathfrak{g}, \omega: \mathfrak{g} \times \mathfrak{g} \rightarrow \mathfrak{g}$ satisfying the closeness condition:

$$
\omega([x, y], z)+\omega([y, z], x)+\omega([z, x], y)=0 \quad \text { for all } x, y, z \in \mathfrak{g} .
$$

The pair $(\mathfrak{g}, \omega)$ is sometimes called a symplectic Lie algebra. 
If $\mathfrak{w}$ is a subspace of $(\mathfrak{g}, \omega)$ the subspace

$$
\mathfrak{w}^{\perp \omega}=\{y \in \mathfrak{g}: \omega(x, y)=0 \quad \text { for all } \quad x \in \mathfrak{w}\}
$$

denotes the symplectic-orthogonal subspace of $\mathfrak{w}$.

In particular we say that $\mathfrak{w}$ is

- isotropic if $\mathfrak{w} \subset \mathfrak{w}^{\perp_{\omega}}$,

- Lagrangian if $\mathfrak{w}=\mathfrak{w}^{\perp \omega}$,

- symplectic if $\mathfrak{w} \cap \mathfrak{w}^{\perp \omega}=0$.

Proposition 4.6. Let $\mathfrak{h}$ be an isotropic ideal on a symplectic Lie algebra $(\mathfrak{g}, \omega)$ then $\mathfrak{h}$ is abelian.

Proof. The closedness condition for $x, y \in \mathfrak{h}, z \in \mathfrak{g}$

$$
0=\omega([x, y], z)+\omega([y, z], x)+\omega([z, x], y)
$$

and since $[y, z] \in \mathfrak{h} \subset \mathfrak{h}^{\perp \omega}$ one gets $\omega([y, z], x)=0$.

Analogously $\omega([z, x], y)=0$. Therefore

$$
0=\omega([x, y], z) \quad \text { for all } z \in \mathfrak{g}
$$

and since $\omega$ is non-degenerate, one has $[x, y]=0$ for all $x, y \in \mathfrak{h}$.

Particular examples arise in the next context. Let $J$ be a complex structure on a symplectic Lie algebra $(\mathfrak{g}, \omega)$. The pair $(\omega, J)$ is called

- a pseudo-Kähler structure on $\mathfrak{g}$ if $\omega(J x, J y)=\omega(x, y)$ for all $x, y \in \mathfrak{g}$.

- a complex-symplectic structure on $\mathfrak{g}$ if $\omega(J x, J y)=-\omega(x, y)$ for all $x, y \in \mathfrak{g}$. See [19] for this definition.

Remark. Notice that there is a one-to-one correspondence between non-degenerate skewsymmetric bilinear maps $\omega$ compatible with an almost complex structure $J$ and metrics $\langle$,$\rangle compatible with an almost complex structure J$ due to

$$
\omega(x, y)=\langle x, J y\rangle \quad \forall x, y \in \mathfrak{g} .
$$

Assume $J$ is integrable. Then if $\nabla$ denotes the Levi-Civita connection associated to $\langle$, then $\nabla J=0$ is equivalent to $d \omega=0$.

Thus for a complex structure $J$ one gets a one-to-one correspondence between

$$
\begin{aligned}
& \left\{\begin{array}{c}
\text { Hermitian structures } \\
\text { such that } \nabla J=0
\end{array}\right\} \quad \longleftrightarrow\{\text { pseudo-Kähler structures for } J\} \\
& \left\{\begin{array}{c}
\text { anti-Hermitian structures } \\
\text { such that } \nabla J=0
\end{array}\right\} \longleftrightarrow\{\text { complex-symplectic structures for } J\}
\end{aligned}
$$

So this equivalence and Proposition 4.4 gives the next result.

Corollary 4.7. Let $(\mathfrak{g}, J)$ be a Lie algebra equipped with an almost complex structure $J$ and assume $\mathfrak{g}$ splits into a direct sum of vector spaces $\mathfrak{g}=\mathfrak{k} \oplus \mathfrak{h}$ such that $J \mathfrak{k}=\mathfrak{h}$. Then $\mathfrak{g}$ always admits a non-degenerate skew-symmetric bilinear map which is compatible or anti-compatible with $J$. 
Example 4.8. Let $\mathfrak{k}$ be a Lie algebra endowed with an (almost) Hermitian structure $\left(B, J_{1}\right)$ and let $\mathfrak{h}$ denote a Lie algebra endowed with an almost Hermitian structure $J_{2}$. Let $\mathfrak{g}=\mathfrak{k} \oplus \mathfrak{h}$ denote the direct sum of vector spaces with complex structure $J:=\left(J_{1}, \pm J_{2}\right)$ as in Remark 3.2. Assume that $\operatorname{dim} \mathfrak{k}=\operatorname{dim} \mathfrak{h}$ and let $t: \mathfrak{k} \rightarrow \mathfrak{h}$ be an isomorphism. If $t J_{1}= \pm J_{2} t$, then the bilinear map $\Omega: \mathfrak{g} \times \mathfrak{g} \rightarrow \mathbb{R}$ given by

$$
\Omega\left(\left(x_{1}, t y_{1}\right),\left(x_{2}, t y_{2}\right)\right)=B\left(x_{1}, J_{1} y_{2}\right)-B\left(x_{2}, J_{1} y_{1}\right)
$$

is skew-symmetric and compatible with $J$.

Proposition 4.9. Let $J$ denote a complex structure on a symplectic Lie algebra $\mathfrak{g}$. Then if $\mathfrak{h}$ is an isotropic ideal, Jh is a Lie subalgebra of $\mathfrak{g}$.

Moreover if $\mathfrak{h}$ is totally real, then $\mathfrak{g}=J \mathfrak{h} \ltimes \mathfrak{h}$.

Proof. The previous proposition says that $\mathfrak{h}$ must be abelian. Now the integrability condition for $J$ gives

$$
[J x, J y]=J[J x, y]+J[x, J y] \quad \text { for all } x, y \in \mathfrak{h}
$$

and $[J x, y]=\pi(J x) y \in \mathfrak{h}$ so as $[x, J y]=-\pi(J y) x \in \mathfrak{h}$ which says that $\mathfrak{k}=J \mathfrak{h}$ is a Lie subalgebra of $\mathfrak{g}$.

If $\mathfrak{h}$ is totally real then $\mathfrak{h} \cap J \mathfrak{h}=\{0\}$ and $\mathfrak{g}=J \mathfrak{h} \ltimes \mathfrak{h}$.

Remark. In the situation of the preceding proposition, if $\mathfrak{h}$ is Lagrangian and the pair $(\omega, j)$ is either pseudo-Kähler or complex-symplectic, then $J \mathfrak{h}$ is a Lagrangian subalgebra.

Let $\mathfrak{k}$ denote a Lie algebra and let $\gamma: \mathfrak{k} \rightarrow \operatorname{End}(\mathfrak{k})$ denote a linear map. Then we say that $\gamma$ is a connection which is

- torsion-free if $\gamma(x) y-\gamma(y) x=[x, y]_{\mathfrak{k}}$

- flat if $\gamma([x, y])=\gamma(x) \gamma(y)-\gamma(y) \gamma(x)$ for all $x, y \in \mathfrak{k}$, that is $\gamma: \mathfrak{k} \rightarrow \operatorname{End}(\mathfrak{k})$ is a representation of Lie algebras.

- Given a symplectic structure on $\mathfrak{k}$ the connection $\gamma$ on $\mathfrak{g}$ is said to be symplectic if

$$
\omega(\gamma(x) y, z)+\omega(y, \gamma(x) z)=0
$$

Lie algebras $\mathfrak{g}$ endowed with a symplectic structure and a torsion-free flat symplectic connection give rise to hypersymplectic structures on $\mathfrak{g} \ltimes V$ where $V$ is the underlying vector space to $\mathfrak{g}$, as proved in [19]. (See proofs and definitions there).

Theorem 4.10. 19] Let $(\mathfrak{g}, \omega)$ be a symplectic Lie algebra with a torsion free, flat symplectic connection $\gamma$ on the underlying vector space $V$ of the Lie algebra. Then the associated space $\mathfrak{g} \ltimes V$ admits an hypersymplectic structure such that the Levi-Civita connection of the associated neutral metric is flat and symplectic with respect to each of the three given symplectic structures.

Generalized complex structures Let $\mathfrak{k}$ be a Lie algebra, and let $\mathfrak{k}^{*}$ denote its dual space. The cotangent Lie algebra $T^{*} \mathfrak{k}$ is the semidirect product of $\mathfrak{k}$ and $\mathfrak{k}^{*}$ via the coadjoint action $\operatorname{ad}^{*}: \mathfrak{k} \rightarrow \operatorname{End}\left(\mathfrak{k}^{*}\right)$, which is given by

$$
\operatorname{ad}^{*}(x) \cdot \varphi=-\varphi \circ \operatorname{ad}(x) \quad \text { for } x \in \mathfrak{k}, \varphi \in \mathfrak{k}^{*} .
$$

The canonical neutral metric on $\mathrm{T}^{*} \mathfrak{k}$ is that one already defined in Example (4.1), also called hyperbolic metric:

$$
\left\langle\left(x_{1}, \varphi_{1}\right),\left(x_{2}, \varphi_{2}\right)\right\rangle=\varphi_{1}\left(x_{2}\right)+\varphi_{2}\left(x_{1}\right) .
$$


According to [4] a generalized complex structure on a Lie algebra $\mathfrak{k}$ is an Hermitian structure $(J,\langle\rangle$,$) on its cotangent Lie algebra \mathrm{T}^{*} \mathfrak{k}$, where $\langle$,$\rangle is the canonical neutral$ metric.

One can see that the dimension of $\mathfrak{k}$ must be even. Assume the dimension of $\mathfrak{k}$ is $2 n$, $\operatorname{dim} \mathfrak{k}=2 n$. If we choose on $\mathrm{T}^{*} \mathfrak{k}$ a basis adapted to the splitting $\mathfrak{k} \oplus \mathfrak{k}^{*}$, the matrix of $J$ has the following form

$$
J=\left(\begin{array}{ll}
j_{1} & j_{2} \\
j_{3} & j_{4}
\end{array}\right)
$$

where $j_{i}$ are certain $2 n \times 2 n$ matrices for $i=1,2,3,4$. One says that $J$ is of type $k$ if rank $j_{2}=2(n-k)$. When $j_{2}=j_{3}=0$ the generalized complex structure $J$ is said to be of complex type; if $j_{1}=j_{4}=0$ the generalized complex structure is called of symplectic type.

If the Lie algebra $\mathfrak{k}$ itself is endowed with a complex structure, then $\mathfrak{k}$ has a generalized complex structure. In fact, if $J_{1}: \mathfrak{k} \rightarrow \mathfrak{k}$ is a complex structure on $\mathfrak{k}$, extending it to $\mathfrak{k}^{*}$ as $J_{2}(\varphi)=-\varphi \circ J_{1}$, the almost complex structure on $\mathrm{T}^{*} \mathfrak{k}$ given by $J(x, \varphi)=\left(J_{1} x, J_{2} \varphi\right)$ is integrable and compatible with the canonical neutral metric. Notice that this is a particular case of (3.2).

Applying results of the previous section we are able to characterize generalized complex structures of symplectic type. In fact, let $J$ denote an Hermitian structure on $\mathrm{T}^{*} \mathfrak{k}$ of symplectic type, then its restriction to $\mathfrak{k}$ induces a linear morphism $j:=J_{\left.\right|_{\mathfrak{k}}}: \mathfrak{k} \rightarrow \mathfrak{k}^{*}$. The integrability of $J$ says that $j$ is a 1 -cocycle of $\left(\mathfrak{k}, \operatorname{ad}^{*}\right)$.

Corollary 4.11. Any generalized complex structure of symplectic type on a even dimensional Lie algebra $\mathfrak{k}$ is determined by a 1-cocycle of $\left(\mathfrak{k}, \mathrm{ad}^{*}\right)$.

Conversely (3.6) and (4.4) imply the following result.

Corollary 4.12. Let $\mathfrak{k}$ be a even dimensional Lie algebra and let $j: \mathfrak{k} \rightarrow \operatorname{End}\left(\mathfrak{k}^{*}\right)$ denote a 1-cocycle of $\left(\mathfrak{k}, \mathrm{ad}^{*}\right)$. Then $j$ induces a generalized complex structure of symplectic type on $\mathrm{T}^{*} \mathfrak{k}$.

Proof. The almost complex structure on $\mathrm{T}^{*} \mathfrak{k}$ given by $J(x, y):=\left(-j^{-1} y, j x\right)$ for $x \in \mathfrak{k}$ $y \in \mathfrak{k}^{*}$ is integrable (see 3.6). Since Proposition 4.4 applies for almost complex structures, the result follows at once.

\section{EXAmples of COMPleX STRUCTURES on SiX Dimensional LiE AlgeBras}

Our goal in this section is to apply Proposition 3.6 to construct complex structures on six dimensional Lie algebras $\mathfrak{g}=\mathfrak{k} \oplus \mathfrak{h}$ where $\mathfrak{h}$ and $\mathfrak{k}$ have the same dimension and $\mathfrak{h}$ is a totally real ideal of $\mathfrak{g}$.

Recall first the classification of real three dimensional Lie algebra $\mathfrak{k}=\operatorname{span}\left\{e_{1}, e_{2}, e_{3}\right\}$, which are listed below (see [27] for instance):

$$
\begin{array}{ll}
\mathbb{R}^{3}: & \text { with trivial Lie bracket } \\
\mathfrak{h}_{1}: & {\left[e_{1}, e_{2}\right]=e_{3} .} \\
\mathfrak{r}_{3} & {\left[e_{1}, e_{2}\right]=e_{2}, \quad\left[e_{1}, e_{3}\right]=e_{2}+e_{3} .} \\
\mathfrak{r}_{3, \lambda}: & {\left[e_{1}, e_{2}\right]=e_{2}, \quad\left[e_{1}, e_{3}\right]=\lambda e_{3} .} \\
\mathfrak{r}_{3, \delta}: & {\left[e_{1}, e_{2}\right]=e_{2}+\delta e_{3} \quad\left[e_{1}, e_{3}\right]=-\delta e_{2}+e_{3} .} \\
\mathfrak{s o}(3): & {\left[e_{1}, e_{2}\right]=e_{3}, \quad\left[e_{1}, e_{3}\right]=-e_{2}, \quad\left[e_{2}, e_{3}\right]=e_{1} .} \\
\mathfrak{s l}(2): & {\left[e_{1}, e_{2}\right]=2, e_{2} \quad\left[e_{1}, e_{3}\right]=2 e_{3}, \quad\left[e_{2}, e_{3}\right]=e_{1} .}
\end{array}
$$


The construction. Let $\mathfrak{k}$ be a three dimensional Lie algebra and let $\mathfrak{h}=\mathbb{R}^{3}$ Let $\pi$ denote a representation from $\mathfrak{k}$ into $\mathfrak{h}$ by derivations. We search for $j \in Z^{1}(\mathfrak{k}, \pi)$ of maximal rank, that is $j: \mathfrak{k} \rightarrow \mathbb{R}^{3}$ is an invertible linear operator satisfying the linear equation

$$
0=\pi(x) j(y)-\pi(y) j(x)-j\left([x, y]_{\mathfrak{k}}\right) \quad \text { for all } x, y \in \mathfrak{k} .
$$

This gives an integrable almost complex structure $J$ on $\mathfrak{g}=\mathfrak{k} \ltimes \mathbb{R}^{3}$, such thar both $\mathfrak{k}$ and $\mathfrak{h}$ as subspaces of $(\mathfrak{g}, J)$ are totally real. The complex structure $J$ on $\mathfrak{g}$ is defined by $J_{\left.\right|_{\mathfrak{k}}}=j, J_{\left.\right|_{\mathfrak{h}}}=j^{-1}$. So if $\mathcal{B}$ is a basis of $\mathfrak{k}$ and $\mathcal{B}^{\prime}$ is a basis of $\mathfrak{h}$, then $\mathcal{B} \cup \mathcal{B}^{\prime}$ is a basis of $\mathfrak{g}$ with respect to which the complex structure $J$ has the form

$$
\left(\begin{array}{cc}
0 & -j^{-1} \\
j & 0
\end{array}\right)
$$

where $j$ is a $3 \times 3$ invertible real matrix. We shall write it with coefficients $j_{u v}$ as follows:

$$
j=\left(\begin{array}{lll}
j_{41} & j_{42} & j_{43} \\
j_{51} & j_{52} & j_{53} \\
j_{61} & j_{62} & j_{63}
\end{array}\right) \quad \operatorname{det} j \neq 0
$$

Next we evaluate the equation (16). If $\left\{e_{1}, e_{2}, e_{3}\right\}$ denotes a basis of $\mathfrak{k}$ and $\left\{e_{4}, e_{5}, e_{6}\right\}$ denotes a basis of $\mathfrak{h}$, Equation (16) becomes

$$
\begin{aligned}
0 & =\pi\left(e_{i}\right) j\left(e_{k}\right)-\pi\left(e_{k}\right) j\left(e_{i}\right)-j\left[e_{i}, e_{k}\right]_{\mathfrak{k}} \\
& =\sum_{s=4}^{6} j_{s k} \pi\left(e_{i}\right) e_{s}-\sum_{s=4}^{6} j_{s i} \pi\left(e_{k}\right) e_{s}-j\left(\sum_{l=1}^{3} C_{i k}^{l}\right) e_{l}
\end{aligned}
$$

which is a linear system on the coefficients $j_{u v}$, for a fixed representation by derivations $\pi$ of $\mathfrak{k}$ into $\mathbb{R}^{3}$ and for $\left\{C_{i k}^{l}\right\}$ being the structure coefficients for $\mathfrak{k}$. We shall choose a representation $\pi$ such that $\operatorname{Im} \pi=1$ to do explicit computations.

For $\mathfrak{h}=\mathbb{R}^{3}$, any representation $\pi: \mathfrak{k} \rightarrow \operatorname{End}\left(\mathbb{R}^{3}\right)$ such that $\operatorname{dim} \operatorname{Im} \pi=1$ is determined by a linear map $t$. Thus there is a basis of $\mathbb{R}^{3}$ in which the matrix of $t$ is of one and only one of the following types

$$
\left.\left.\left.i)\left(\begin{array}{ccc}
\eta & 0 & 0 \\
0 & \nu & 0 \\
0 & 0 & \mu
\end{array}\right) \quad i i\right)\left(\begin{array}{ccc}
\eta & 0 & 0 \\
0 & \nu & -\mu \\
0 & \mu & \nu
\end{array}\right) \quad i i i\right)\left(\begin{array}{ccc}
\eta & 0 & 0 \\
0 & \nu & 1 \\
0 & 0 & \nu
\end{array}\right) \quad i v\right)\left(\begin{array}{ccc}
\eta & 1 & 0 \\
0 & \eta & 1 \\
0 & 0 & \eta
\end{array}\right)
$$

For $\mathfrak{h}=\mathfrak{h}_{1}$, any representation $\pi: \mathfrak{k} \rightarrow \operatorname{Der}\left(\mathfrak{h}_{1}\right)$ such that $\operatorname{dim} \operatorname{Im} \pi=1$ is determined by a derivation $t$ of $\mathfrak{h}_{1}$. Thus there is a basis of $\mathfrak{h}_{1}$ in which the matrix of $t$ is of one and only one of the following types (see for instance [5]):

$$
\text { i) }\left(\begin{array}{ccc}
\eta & \nu & 0 \\
\mu & -\eta & 0 \\
0 & 0 & 0
\end{array}\right) \quad \text { ii) }\left(\begin{array}{ccc}
\eta & \nu & 0 \\
\mu & 1-\eta & 0 \\
0 & 0 & 1
\end{array}\right)
$$

In any case if we assume that $\pi\left(e_{1}\right)=\varepsilon_{1} t, \pi\left(e_{2}\right)=\varepsilon_{2} t, \pi\left(e_{3}\right)=\varepsilon_{3} t$, the condition of $\pi$ being a representation says

$$
\phi([x, y])=[\pi(x), \pi(y)]=0
$$

where the last equality holds due to $\operatorname{Im} \pi=\operatorname{span}\{t\}$. Hence $\pi(x)=0$ for every $x \in C^{1}(\mathfrak{k})$. 
This explanation gives the proof of the following Lemma. See for instance [33] for representations of $\mathfrak{s l}(2)$.

Lemma 5.1. The simple Lie algebras $\mathfrak{s l}(2)$ and $\mathfrak{s o}(3)$ do not admit any representation $\pi: \mathfrak{k} \rightarrow \operatorname{End}(V)$ such that $\operatorname{dim} \operatorname{Im} \pi=1$.

For the solvable Lie algebras $\mathfrak{h}_{1}, \mathfrak{r}_{3}, \mathfrak{r}_{3, \lambda}, \mathfrak{r}_{3, \delta}$ one has $\pi\left(e_{j}\right)=0$ for $e_{j} \in C^{1}(\mathfrak{k})$. Since in all these cases $e_{1} \in \mathfrak{k}-C^{1}(\mathfrak{k})$ we shall assume $\pi\left(e_{1}\right)=t$ and we take $\pi\left(e_{2}\right)=\varepsilon_{1} t, \pi\left(e_{3}\right)=\varepsilon_{2} t$, where $\varepsilon_{i}$ could be zero as explained above.

The computations for the proof of the next theorems can be done with help of a software.

Theorem 5.2. Let $\mathfrak{k}$ be a three dimensional solvable Lie algebra. Let $\pi: \mathfrak{k} \rightarrow \operatorname{End}\left(\mathbb{R}^{3}\right)$ be a representation such that $\operatorname{Im} \pi=\operatorname{span}\{t\}$ where $t$ is as in (17). Then the semidirect product Lie algebra $\mathfrak{g}=\mathfrak{k} \oplus_{\pi} \mathbb{R}^{3}$ admits a complex structure $J$ such that $J \mathfrak{k}=\mathbb{R}^{3}$ in the cases exposed in Table 1.

Theorem 5.3. Let $\mathfrak{k}$ be a three dimensional solvable Lie algebra. Let $\pi: \mathfrak{k} \rightarrow \operatorname{End}\left(\mathfrak{h}_{1}\right)$ be a representation acting by derivations on the Heisenberg Lie algebra $\mathfrak{h}_{1}$ with $\operatorname{dim} \operatorname{Im} \pi=1$ as in (18).

Then there exists a Lie algebra with a complex structure $(\mathfrak{g}, J)$ such that $\mathfrak{h}_{1}$ is a totally real ideal of the extended semidirect product Lie algebra $\mathfrak{g}=\mathfrak{k} \oplus_{\pi} \mathfrak{h}_{1}$ as in Proposition [3.6 in the cases exposed in Table 2.

Table 1

\begin{tabular}{|c|c|c|c|}
\hline $\mathfrak{k}$ & Type $t$ & Existence results & Parameters of $t$ for the existence \\
\hline $\mathfrak{h}_{1}$ & (i) & no & \\
\hline & (ii) & no & $\nu=0$ \\
\hline & (iii) & yes & $\eta=0$ \\
\hline & (iv) & yes & \\
\hline $\mathfrak{r}_{3}$ & (i) & no & $\nu=1$ \\
\hline & (ii) & no & $\eta=1$ \\
\hline & (iii) & yes & $\eta=\nu=0, \mu=1, \varepsilon=0$ \\
\hline & (iv) & yes & $\eta=\mu, \nu=1, \varepsilon=0$ \\
\hline $\mathfrak{r}_{3, \lambda}$ & (i) & yes & $\eta=1, \nu=\mu=0, \varepsilon=0$ \\
$\lambda=0$ & & & $\nu=1, \mu=0, \varepsilon=0$ \\
& & & $\nu=0, \mu=1, \varepsilon=0$ \\
& & & $\eta=1, \nu=0, \varepsilon=0$ \\
& & & $\eta=1, \mu=0, \varepsilon=0$ \\
& & & $\eta=0, \mu=1, \varepsilon=0$ \\
& & & $\eta=0, \nu=1, \varepsilon=0$ \\
& & & $\eta=, \mu=\nu=1 \varepsilon=0$ \\
& & & $\eta=1, \nu=0, \mu=1, \varepsilon=0$ \\
& & & $\eta=1, \nu=1, \mu=0, \varepsilon=0$ \\
& & &
\end{tabular}




\begin{tabular}{|c|c|c|c|}
\hline $\bar{k}$ & Type $t$ & Existence results & Parameters of $t$ for the existence \\
\hline & (ii) & yes & $\begin{array}{l}\eta=1, \nu=\mu=\varepsilon=0 \\
\nu=1, \eta=\mu=\varepsilon=0\end{array}$ \\
\hline & (iii) & yes & $\begin{array}{l}\eta=1, \nu=\varepsilon=0 \\
\nu=1, \eta=\varepsilon=0\end{array}$ \\
\hline & (iv) & no & \\
\hline $\begin{array}{l}\mathfrak{r}_{3, \lambda} \\
\lambda \neq 0\end{array}$ & (i) & yes & $\begin{array}{c}\eta=1, \mu=\lambda \\
\eta=1, \nu=\lambda \\
\eta=\lambda, \nu=1 \\
\eta=\lambda, \mu=1 \\
\nu=1, \mu=\lambda \\
\nu=\lambda, \mu=1 \\
\eta=1, \nu=\mu=\lambda \\
\eta=\nu=1, \mu=\lambda \\
\eta=\mu=1, \nu=\lambda \\
\eta=\nu=\lambda, \mu=1 \\
\eta=\mu=\lambda, \nu=1 \\
\eta=\lambda, \nu=\mu=1 \\
\lambda=\eta=\nu=1 \\
\lambda=\eta=\mu=1 \\
\lambda=\nu=\mu=1 \\
\lambda=\eta=\nu=\mu=1\end{array}$ \\
\hline & (ii) & yes & $\begin{array}{c}\lambda=\nu=1, \mu=0 \\
\eta=1, \nu=\lambda, \mu=0 \\
\eta=\lambda, \nu=1, \mu=0\end{array}$ \\
\hline & (iii) & yes & $\begin{array}{c}\eta=1, \nu=\lambda \\
\eta=\lambda, \nu=1 \\
\lambda=\eta=\nu=1\end{array}$ \\
\hline & (iv) & no & \\
\hline $\mathfrak{r}_{3, \delta}$ & (i) & yes & $\begin{array}{c}\delta=0, \nu=0, \mu=1 \\
\delta=0, \eta=1, \nu=0\end{array}$ \\
\hline & (ii) & yes & $\begin{array}{c}\nu=1, \mu=\delta \\
\nu=1, \mu=-\delta\end{array}$ \\
\hline & (iii) & yes & $\delta=0, \eta=\nu=1$ \\
\hline & (iv) & no & \\
\hline
\end{tabular}

Table 2

\begin{tabular}{|r|c|c|c|}
\hline $\mathfrak{k}$ & Type $t$ & Existence results & Parameters of $t$ for the existence \\
\hline $\mathfrak{h}_{1}$ & (i) & yes & $\begin{array}{c}\nu \neq 0, \mu=-\frac{\eta^{2}}{\nu} \\
\eta=\nu=0\end{array}$ \\
\hline & (ii) & no & \\
\hline $\mathfrak{r}_{3}$ & (i) & no & \\
\hline $\mathfrak{r}_{3}$ & (ii) & no & \\
\hline
\end{tabular}




\begin{tabular}{|c|c|c|c|}
\hline $\mathfrak{k}$ & Type $t$ & Existence results & Parameters of $t$ for the existence \\
\hline \multirow[t]{2}{*}{$\mathfrak{r}_{3, \lambda}$} & (i) & yes & $\begin{array}{c}\lambda=0, \nu \neq 0, \varepsilon=0 \\
\lambda=0, \eta=1, \nu=0, \varepsilon=0 \\
\lambda=0, \eta=-1, \nu=0, \varepsilon=0 \\
\lambda=-1, \eta=1, \mu=0 \\
\lambda=-1, \eta=\frac{j_{43} \mu+j_{53}}{j_{53}^{2}}, \nu=-\frac{\left(j_{43} \mu+2 j_{53}\right) j_{43}}{j_{53}^{2}} \\
\lambda=-1, \eta=-1, \nu=2 \frac{j_{42}}{j_{52}}, \mu=0\end{array}$ \\
\hline & (ii) & yes & $\begin{array}{c}\lambda=0, \nu \neq 0, \mu=-\frac{\eta(\eta-1)}{\nu}, \varepsilon=0 \\
\lambda=0, \eta=1, \nu=0, \varepsilon=0 \\
\lambda=0, \eta=\nu=0, \varepsilon=0 \\
\lambda=0, \eta=\mu=0, \varepsilon=0 \\
\lambda=0, \eta=\nu=0, \mu=-\frac{j_{53}}{j_{43}}, \varepsilon=0 \\
\lambda=1, \nu \neq 0, \mu=-\frac{\eta(\eta-1)}{\nu} \\
\lambda=1, \eta=1, \nu=0 \\
\lambda=1, \eta=\mu=0 \\
\lambda=1, \eta=\nu=0 \\
\lambda \neq 0, \eta=\frac{\mu j_{43}-\lambda j_{53}+j_{53}}{j_{53}}, \nu=-\frac{j_{43}\left(\mu j_{43}-2 \lambda j_{53}+j_{53}\right)}{j_{53}^{2}} \\
\lambda \neq 0, \eta=\lambda, \mu=0\end{array}$ \\
\hline \multirow[t]{2}{*}{$\mathfrak{r}_{3, \delta}$} & (i) & no & \\
\hline & (ii) & yes & $\begin{array}{c}\delta=0, \nu \neq 0, \mu=-\frac{\eta(\eta-1)}{\nu} \\
\delta=0, \eta=1, \nu=0 \\
\delta=0, \eta=\mu=0 \\
\delta=0, \eta=\nu=0\end{array}$ \\
\hline
\end{tabular}

The explicit matrix realizations of $j: \mathfrak{k} \rightarrow \mathbb{R}^{3}$ and $j: \mathfrak{k} \rightarrow \mathfrak{h}_{1}$ can be seen in [17.

Acknoledgements. RCS acknowledges partial support from the research project MTM201018556 of the MICINN (Spain).

I. Cardoso and G. Ovando were partially supported by SCyT-UNR and CONICET.

\section{REFERENCES}

[1] A. L. Agore, G. Militaru, Extending structures for Lie algebras, Monatsh. Math. DOI:10.1007/s00605-013-0537-7 (2013), (arXiv:math.RA 1301.5442). 2

[2] A. Andrada, M. L. Barberis, I. G. Dotti, Classification of abelian complex structures on six-dimensional Lie algebras, J. London Math. Soc. 83, 231-255 (2011). 1] 2

[3] A. Andrada, M. L. Barberis, I. G. Dotti, Abelian Hermitian geometry, Diff. geom. Appl. 30 (5), 509-519 (2012). 2

[4] L. de Andres, M. L. Barberis, I. G. Dotti, M. Fernandez, Hermitian structures on cotangent bundles of four dimensional solvable Lie groups, Osaka J. Math 44 (4), 765-793 (2007). 2 [16]

[5] A. Andrada, M. L. Barberis, I. G. Dotti, G. P. Ovando, Product structures on four dimensional solvable Lie algebras. Homology Homotopy and Applications 7, 9-37 (2005). 6. 17

[6] A. Andrada, M. L. Barberis, G. P. Ovando, Lie bialgebras of complex type and associated Poisson Lie groups, J. Geom. Phys. 58, 1310-1328 (2008). 13

[7] H. Anciaux, P. Romon, A canonical structure on the tangent bundle of a pseudo-Kähler or para-Kähler manifold, to appear Monatsh. Math. (arXiv.mathDG: 1301.4638). 2

[8] A. Andrada, S. Salamon, Complex product structures on Lie algebras, Forum Math. 17 (2), 261-295 (2005). 2, 10 
[9] O. Baues, V. Cortes, Symplectic Lie groups, arXiv:math.DG: 1307.1629v2. 2

[10] M. L. BARBERIS, A. Fino, New HKT manifolds arising from quaternionic representations, Math. Z. 267 (3-4), 717-735 (2011). 2

[11] N. BlažIĆ, S. ; Vukmirović, Four-dimensional Lie algebras with a para-hypercomplex structure Rocky Mt. J. Math. 40 (5), 1391-1439 (2010). 10

[12] M. Bordemann, Nondegenerate invariant bilinear forms on nonassociative algebras, Acta Math. Univ. Comenianae LXVI (2), 151-201 (1997).

[13] C. Chevalley, S. Eilenberg, Cohomology theory of Lie groups and Lie algebras, Trans. Amer. Math. Soc., 63, 85-124 (1948). 2

[14] G. Calvaruso, A. Fino, Complex and paracomplex structures on homogeneous pseudoRiemannian four-manifolds, Int. J. Math. 24 (1), Paper No. 1250130, 28 p. (2013). 1

[15] V. Cruceanu, P. Fortuny, P. M. Gadea, A survey on paracomplex geometry. Rocky Mount. J. Math. 26 (1), 83-115 (1996). 2, 6

[16] R. Cleyton, J. Lauret, Y. S. Poon, Weal mirror symmetry of Lie algebras, J. Symplectic Geom. 8, 37-55 (2010). 10

[17] R. Campoamor Stursberg, I. E. Cardoso, G. P. Ovando, Addedum to "Extending complex structures". 20

[18] R. Campoamor Stursberg, G. P. Ovando, Complex structures on tangent and cotangent Lie algebras, Osaka J. Math. 49 (2), 489-513 (2012). 1, 10

[19] R. Cleyton, G. Ovando, Y.S.Poon, Weak mirror symmetry of complex symplectic Lie algebras, J. Geom. Phys. 61, 1553-1563 (2011). 14, 15

[20] M. Ceballos, A. Otal, L. Ugarte, R. Villacampa, Classification of complex structures on 6-dimensional nilpotent Lie algebras, arXiv:math.DG:1111.5873v4. 1

[21] N. Enrietti, A. Fino, Special Hermitian metrics and Lie groups, Differ. Geom. Appl. 29 Suppl. 1, 211-219 (2011). 1, 13

[22] N. Enrietti, A. Fino, L. Vezzoni, Tamed symplectic forms and SKT metrics, J. Symplectic Geom. 10 (2), 203-223 (2012). 2

[23] A. Fino, H. Kasuya, L. Vezzoni, Tamed complex structures on solvmanifolds, arXiv:math.DG 1302.5569. 2

[24] A. Fino, A. Otal, L. UGarte, Six dimensional solvmanifolds with holomorphically trivial canonical bundle, arXiv.math.DG 1401.0512. 1

[25] L. Garca Vergnolle, E. Remm, Complex structures on quasi-filiform Lie algebras, J. Lie Theory 19 (2), 251-265 (2009). 2

[26] M. Goze, E. Remm, On the non existence of complex structures on filiform nilpotent Lie algebras, Comm. Alg., 30 (8), 3777-3788 (2002). 2

[27] J. Milnor, Curvatures of left invariant metrics on Lie groups, Advances in Mathematics, 21, 293-329 (1976). 16

[28] A. P. Norden, On a class of four dimensional A-spaces Russian Math. (Izv. VUZ) 17, 145-157 (1960). 11

[29] G. OvANDO, Invariant complex structures on solvable real Lie groups, Manuscripta Math. 103, 19-30 (2000). 1 6

[30] G. Ovando, Complex, symplectic and Kähler structures on four dimensional Lie groups, Rev. Unión Mat. Argent. 45 (2), 55-67 (2004). 1

[31] S. Rollenske, Geometry of nilmanifolds with left invariant complex structures and deformations in the large, Proc. London Math. Soc. 99, 425-460 (2009). 9

[32] J. E. SNOw, Invariant complex structures on four-dimensional solvable real Lie groups, Manuscripta Math. 66 (4), 397-412 (1990). 1, 6

[33] V.S. VARAdARAJAn, Lie Groups, Lie Algebras, and Their Representations, Springer (1984). 18] 
R. Campoamor Stursberg: I.M.I and Depto. Geometra y Topologa, Universidad ComPLUTENSE DE MADRID, Spain

E-mail address: rutwig@ucm.es

I. E. Cardoso, ECEn-FCeia, Universidad Nacional de Rosario, Pellegrini 250, 2000 Rosario, Santa Fe, Argentina

E-mail address: isolda@fceia.unr.edu.ar

G. P. Ovando: COniCET and ECEn-FCEia, Universidad Nacional de Rosario, Pellegrini 250, 2000 Rosario, Santa Fe, Argentina

E-mail address: gabriela@fceia.unr.edu.ar 


\title{
ADDENDUM TO: "EXTENDING INVARIANT COMPLEX STRUCTURES"
}

\author{
RUTWIG CAMPOAMOR STURSBERG, ISOLDA E. CARDOSO, AND GABRIELA P. OVANDO
}

In this note we write the explicit computations given in the work "Extending complex structures" (by R. Campoamor Stursberg, I. E. Cardoso and G. P. Ovando) in the Section Examples of totally real complex structures on six dimensional Lie algebras.

The goal is to construct complex structures on six dimensional Lie algebras $\mathfrak{g}=\mathfrak{k} \oplus \mathfrak{h}$ where $\mathfrak{h}$ is an ideal of $\mathfrak{g}$ isomorphic to $\mathbb{R}^{3}$ and $\mathfrak{k}$ is a subalgebra of dimension three.

Recall first the classification of real three dimensional Lie algebra $\mathfrak{k}=\operatorname{span}\left\{e_{1}, e_{2}, e_{3}\right\}$, which are listed below (see 2] for instance):

$$
\begin{array}{lll}
\mathbb{R}^{3}: & \text { with trivial Lie bracket } \\
\mathfrak{h}_{1}: & {\left[e_{1}, e_{2}\right]=e_{3} .} \\
\mathfrak{r}_{3} & {\left[e_{1}, e_{2}\right]=e_{2}, \quad\left[e_{1}, e_{3}\right]=e_{2}+e_{3} .} \\
\mathfrak{r}_{3, \lambda}: & {\left[e_{1}, e_{2}\right]=e_{2}, \quad\left[e_{1}, e_{3}\right]=\lambda e_{3} .} \\
\mathfrak{r}_{3, \delta}: & {\left[e_{1}, e_{2}\right]=e_{2}+\delta e_{3} \quad\left[e_{1}, e_{3}\right]=-\delta e_{2}+e_{3} .} \\
\mathfrak{s o}(3): & {\left[e_{1}, e_{2}\right]=e_{3}, \quad\left[e_{1}, e_{3}\right]=-e_{2}, \quad\left[e_{2}, e_{3}\right]=e_{1} .} \\
\mathfrak{s l}(2): & {\left[e_{1}, e_{2}\right]=2, e_{2} \quad\left[e_{1}, e_{3}\right]=2 e_{3}, \quad\left[e_{2}, e_{3}\right]=e_{1} .}
\end{array}
$$

The construction. Let $\mathfrak{k}$ be a three dimensional Lie algebra and let $\mathfrak{h}=\mathbb{R}^{3}$ Let $\pi$ denote a representation from $\mathfrak{k}$ into $\mathfrak{h}$ by derivations. We search for $j \in Z^{1}(\mathfrak{k}, \pi)$ of maximal rank, that is $j: \mathfrak{k} \rightarrow \mathbb{R}^{3}$ is an invertible linear operator satisfying the linear equation

$$
0=\pi(x) j(y)-\pi(y) j(x)-j\left([x, y]_{\mathfrak{k}}\right) \quad \text { for all } x, y \in \mathfrak{k} .
$$

This gives an integrable almost complex structure $J$ on $\mathfrak{g}=\mathfrak{k} \ltimes \mathbb{R}^{3}$, such thar both $\mathfrak{k}$ and $\mathfrak{h}$ as subspaces of $(\mathfrak{g}, J)$ are totally real. The complex structure $J$ on $\mathfrak{g}$ is defined by $J_{\left.\right|_{\mathfrak{k}}}=j, J_{\left.\right|_{\mathfrak{h}}}=j^{-1}$. So if $\mathcal{B}$ is a basis of $\mathfrak{k}$ and $\mathcal{B}^{\prime}$ is a basis of $\mathfrak{h}$, then $\mathcal{B} \cup \mathcal{B}^{\prime}$ is a basis of $\mathfrak{g}$ with respect to which the complex structure $J$ has the form

$$
\left(\begin{array}{cc}
0 & -j^{-1} \\
j & 0
\end{array}\right)
$$

where $j$ is a $3 \times 3$ invertible real matrix. We shall write it with coefficients $j_{u v}$ as follows:

$$
j=\left(\begin{array}{lll}
j_{41} & j_{42} & j_{43} \\
j_{51} & j_{52} & j_{53} \\
j_{61} & j_{62} & j_{63}
\end{array}\right) \quad \operatorname{det} j \neq 0 .
$$

Next we evaluate the equation (11). If $\left\{e_{1}, e_{2}, e_{3}\right\}$ denotes a basis of $\mathfrak{k}$ and $\left\{e_{4}, e_{5}, e_{6}\right\}$ denotes a basis of $\mathfrak{h}$, Equation (10) becomes

$$
\begin{aligned}
0 & =\pi\left(e_{i}\right) j\left(e_{k}\right)-\pi\left(e_{k}\right) j\left(e_{i}\right)-j\left[e_{i}, e_{k}\right]_{\mathfrak{k}} \\
& =\sum_{s=4}^{6} j_{s k} \pi\left(e_{i}\right) e_{s}-\sum_{s=4}^{6} j_{s i} \pi\left(e_{k}\right) e_{s}-j\left(\sum_{l=1}^{3} C_{i k}^{l}\right) e_{l}
\end{aligned}
$$

which is a linear system on the coefficients $j_{u v}$, for a fixed representation by derivations $\pi$ of $\mathfrak{k}$ into $\mathbb{R}^{3}$ and for $\left\{C_{i k}^{l}\right\}$ being the structure coefficients for $\mathfrak{k}$. We shall choose a representation $\pi$ such that $\operatorname{Im} \pi=1$ to do explicit computations.

For $\mathfrak{h}=\mathbb{R}^{3}$, any representation $\pi: \mathfrak{k} \rightarrow \operatorname{End}\left(\mathbb{R}^{3}\right)$ such that $\operatorname{dim} \operatorname{Im} \pi=1$ is determined by a linear map $t$. Thus there is a basis of $\mathbb{R}^{3}$ in which the matrix of $t$ is of one and only one of the following types 

i) $\left(\begin{array}{lll}\eta & 0 & 0 \\ 0 & \nu & 0 \\ 0 & 0 & \mu\end{array}\right)$
ii) $\left(\begin{array}{ccc}\eta & 0 & 0 \\ 0 & \nu & -\mu \\ 0 & \mu & \nu\end{array}\right)$
iii) $\left(\begin{array}{lll}\eta & 0 & 0 \\ 0 & \nu & 1 \\ 0 & 0 & \nu\end{array}\right)$
$i v)\left(\begin{array}{lll}\eta & 1 & 0 \\ 0 & \eta & 1 \\ 0 & 0 & \eta\end{array}\right)$.

For $\mathfrak{h}=\mathfrak{h}_{1}$, any representation $\pi: \mathfrak{k} \rightarrow \operatorname{Der}\left(\mathfrak{h}_{1}\right)$ such that $\operatorname{dim} \operatorname{Im} \pi=1$ is determined by a derivation $t$ of $\mathfrak{h}_{1}$. Thus there is a basis of $\mathfrak{h}_{1}$ in which the matrix of $t$ is of one and only one of the following types (see for instance [1]):
i) $\left(\begin{array}{ccc}\eta & \nu & 0 \\ \mu & -\eta & 0 \\ 0 & 0 & 0\end{array}\right)$
ii) $\left(\begin{array}{ccc}\eta & \nu & 0 \\ \mu & 1-\eta & 0 \\ 0 & 0 & 1\end{array}\right)$.

In any case if we assume that $\pi\left(e_{1}\right)=\varepsilon_{1} t, \pi\left(e_{2}\right)=\varepsilon_{2} t, \pi\left(e_{3}\right)=\varepsilon_{3} t$, the condition of $\pi$ being a representation says

$$
\phi([x, y])=[\pi(x), \pi(y)]=0
$$

where the last equality holds due to $\operatorname{Im} \pi=\operatorname{span}\{t\}$. Hence $\pi(x)=0$ for every $x \in C^{1}(\mathfrak{k})$.

This explanation gives the proof of the following Lemma. See for instance $[3]$ for representations of $\mathfrak{s l}(2)$.

Lemma 0.1. The simple Lie algebras $\mathfrak{s l}(2)$ and $\mathfrak{s o}(3)$ do not admit any representation $\pi: \mathfrak{k} \rightarrow$ $\operatorname{End}(V)$ such that $\operatorname{dim} \operatorname{Im} \pi=1$.

For the solvable Lie algebras $\mathfrak{h}_{1}, \mathfrak{r}_{3}, \mathfrak{r}_{3, \lambda}, \mathfrak{r}_{3, \delta}$ one has $\pi\left(e_{j}\right)=0$ for $e_{j} \in C^{1}(\mathfrak{k})$. Since in all these cases $e_{1} \in \mathfrak{k}-C^{1}(\mathfrak{k})$ we shall assume $\pi\left(e_{1}\right)=t$ and we take $\pi\left(e_{2}\right)=\varepsilon_{1} t, \pi\left(e_{3}\right)=\varepsilon_{2} t$, where $\varepsilon_{i}$ could be zero as explained above.

The computations for the proof of the next theorems can be done with help of a software.

Theorem 0.2. Let $\mathfrak{k}$ be a three dimensional solvable Lie algebra. Let $\pi: \mathfrak{k} \rightarrow \operatorname{End}\left(\mathbb{R}^{3}\right)$ be a representation such that $\operatorname{Im} \pi=\operatorname{span}\{t\}$ where $t$ is as in (2). Then the semidirect product Lie algebra $\mathfrak{g}=\mathfrak{k} \oplus_{\pi} \mathbb{R}^{3}$ admits a complex structure $J$ such that $J \mathfrak{k}=\mathbb{R}^{3}$ in the cases exposed in Table 1.

Theorem 0.3. Let $\mathfrak{k}$ be a three dimensional solvable Lie algebra. Let $\pi: \mathfrak{k} \rightarrow \operatorname{End}\left(\mathfrak{h}_{1}\right)$ be a representation acting by derivations on the Heisenberg Lie algebra $\mathfrak{h}_{1}$ with $\operatorname{dim} \operatorname{Im} \pi=1$ as in (3).

Then there exists a Lie algebra with a complex structure $(\mathfrak{g}, J)$ such that $\mathfrak{h}_{1}$ is a totally real ideal of the extended semidirect product Lie algebra $\mathfrak{g}=\mathfrak{k} \oplus_{\pi} \mathfrak{h}_{1}$ (as in Proposition 3.6 of "Extending complex structures") in the cases exposed in Table 2.

The results. Next we present the results of these computations done as follows: for each Lie algebra $\mathfrak{h}=\mathbb{R}^{3}$ and $\mathfrak{h}=\mathfrak{h}_{1}$, and for each Lie algebra $\mathfrak{k}$ (cases I to IV) we show the solutions, if there are solutions, for each type of representation (types i to iv). Each solution consists of a matrix with two horizontal entries: the above entry shows the form of the matrix $j$ and its determinant, which we consider nonzero, and the below entry shows the parameters $\eta, \nu, \mu, \varepsilon_{1}, \varepsilon_{2}, \lambda$ and $\delta$, wherever they correspond. Also, we remark that some of the solutions are complex, which we do not consider as a valid solution for our problem but we present them anyway for the sake of completeness. Finally, below the explicit solutions we present the table, depicted in the article, where we summarize this computations.

0.1. Computations for $\mathfrak{h}=\mathbb{R}^{3}$.

(1) CASE I: $\mathfrak{k}=\mathfrak{h}_{1}$.

(a) For a type (i) representation: there are no solutions.

(b) For a type (ii) representation: there are no solutions. 
(c) For a type (iii) there are 2 solutions.

$$
\left.\begin{array}{c}
{\left[j=\left[\begin{array}{ccc}
j_{41} & j_{42} & 0 \\
j_{51} & j_{52} & j_{53} \\
j_{61} & j_{53} & 0
\end{array}\right], \operatorname{det}(j)=-j_{41} j_{53}{ }^{2}+j_{42} j_{53} j_{61}\right]} \\
{[\eta=0, \nu=0, \varepsilon=\varepsilon]}
\end{array}\right]
$$

(d) For a type (iv) representation: there is 1 solution.

$$
\left[\begin{array}{c}
{\left[j=\left[\begin{array}{ccc}
j_{41} & j_{42} & j_{43} \\
j_{51} & \varepsilon j_{51}+j_{43} & 0 \\
j_{61} & \varepsilon j_{61} & 0
\end{array}\right], \operatorname{det}(j)=-j_{43}{ }^{2} j_{61}\right]} \\
{[\eta=0, \varepsilon=\varepsilon]}
\end{array}\right.
$$

(2) CASE II: $\mathfrak{k}=\mathfrak{r}_{3}$.

(a) For a type (i) representation: there are no solutions.

(b) For a type (ii) representation: there are no solutions.

(c) For a type (iii) there are 2 solutions.

$$
\left.\begin{array}{c}
{\left[j=\left[\begin{array}{ccc}
j_{41} & 0 & j_{43} \\
j_{51} & j_{52} & j_{53} \\
j_{61} & 0 & j_{52}
\end{array}\right], \operatorname{det}(j)=j_{41} j_{52}{ }^{2}-j_{61} j_{43} j_{52}\right]} \\
{[\eta=1, \nu=1]} \\
{\left[j=\left[\begin{array}{ccc}
j_{41} & 0 & 0 \\
j_{51} & j_{52} & j_{53} \\
j_{61} & 0 & j_{52}
\end{array}\right], \operatorname{det}(j)=j_{41} j_{52}{ }^{2}\right]} \\
{[\eta=\eta, \nu=1]}
\end{array}\right]
$$

(d) For a type (iv) there is 1 solution.

$$
\left[\begin{array}{c}
{\left[j=\left[\begin{array}{ccc}
j_{41} & j_{53} & j_{43} \\
j_{51} & 0 & j_{53} \\
j_{61} & 0 & 0
\end{array}\right], \operatorname{det}(j)=j_{53}{ }^{2} j_{61}\right]} \\
{[\eta=1]}
\end{array}\right.
$$

(3) CASE III: $\mathfrak{k}=\mathfrak{r}_{3, \lambda}$.

(a) For a type (i) representation: there are 28 solutions.

$$
\left.\begin{array}{c}
{\left[j=\left[\begin{array}{ccc}
j_{41} & 0 & j_{43} \\
j_{51} & 0 & j_{53} \\
j_{61} & j_{62} & 0
\end{array}\right], \operatorname{det}(j)=-j_{41} j_{53} j_{62}+j_{43} j_{51} j_{62}\right]} \\
{[\lambda=0, \eta=0, \nu=0, \mu=1, \varepsilon=0]}
\end{array}\right]
$$


R. CAMPOAmOR StuRsBerG, I. E. CARDOSO, AND G. P. OVANDO

$$
\begin{aligned}
& \begin{array}{c}
{\left[j=\left[\begin{array}{ccc}
j_{41} & 0 & j_{43} \\
j_{51} & j_{52} & 0 \\
j_{61} & 0 & j_{63}
\end{array}\right], \operatorname{det}(j)=j_{41} j_{52} j_{63}-j_{43} j_{52} j_{61}\right]} \\
{[\lambda=0, \eta=0, \nu=1, \mu=0, \varepsilon=0]}
\end{array} \\
& \left.\begin{array}{c}
{\left[j=\left[\begin{array}{ccc}
j_{41} & j_{42} & 0 \\
j_{51} & 0 & j_{53} \\
j_{61} & 0 & j_{63}
\end{array}\right], \operatorname{det}(j)=-j_{42} j_{51} j_{63}+j_{42} j_{53} j_{61}\right]} \\
{[\lambda=0, \eta=1, \nu=0, \mu=0, \varepsilon=0]}
\end{array}\right] \\
& \left.\begin{array}{c}
{\left[j=\left[\begin{array}{ccc}
j_{41} & 0 & 0 \\
j_{51} & j_{52} & 0 \\
j_{61} & 0 & j_{63}
\end{array}\right], \operatorname{det}(j)=j_{41} j_{52} j_{63}\right]} \\
{[\lambda=0, \eta=\eta, \nu=1, \mu=0, \varepsilon=0]}
\end{array}\right] \\
& \left.\begin{array}{c}
{\left[j=\left[\begin{array}{ccc}
j_{41} & 0 & 0 \\
j_{51} & 0 & j_{53} \\
j_{61} & j_{62} & 0
\end{array}\right], \operatorname{det}(j)=-j_{41} j_{53} j_{62}\right]} \\
{[\lambda=0, \eta=\eta, \nu=0, \mu=1, \varepsilon=0]}
\end{array}\right] \\
& \left.\begin{array}{c}
{\left[\left[\begin{array}{ccc}
j_{41} & j_{42} & 0 \\
j_{51} & 0 & 0 \\
j_{61} & 0 & j_{63}
\end{array}\right], \operatorname{det}(j)=-j_{42} j_{51} j_{63}\right]} \\
{[\lambda=0, \eta=1, \nu=\nu, \mu=0, \varepsilon=0]}
\end{array}\right] \\
& \left.\begin{array}{c}
{\left[j=\left[\begin{array}{ccc}
j_{41} & j_{42} & 0 \\
j_{51} & 0 & j_{53} \\
j_{61} & 0 & 0
\end{array}\right], \operatorname{det}(j)=j_{42} j_{53} j_{61}\right]} \\
{[\lambda=0, \eta=1, \nu=0, \mu=\mu, \varepsilon=0]}
\end{array}\right] \\
& \left.\begin{array}{c}
{\left[j=\left[\begin{array}{ccc}
j_{41} & 0 & j_{43} \\
j_{51} & 0 & 0 \\
j_{61} & j_{62} & 0
\end{array}\right], \operatorname{det}(j)=j_{43} j_{51} j_{62}\right]} \\
{[\lambda=0, \eta=0, \nu=\nu, \mu=1, \varepsilon=0]}
\end{array}\right] \\
& \left.\begin{array}{c}
{\left[j=\left[\begin{array}{ccc}
j_{41} & 0 & j_{43} \\
j_{51} & j_{52} & 0 \\
j_{61} & 0 & 0
\end{array}\right], \operatorname{det}(j)=-j_{43} j_{52} j_{61}\right]} \\
{[\lambda=0, \eta=0, \nu=1, \mu=\mu, \varepsilon=0]}
\end{array}\right] \\
& \left.\begin{array}{c}
{\left[j=\left[\begin{array}{ccc}
j_{41} & j_{42} & 0 \\
j_{51} & 0 & j_{53} \\
j_{61} & j_{62} & 0
\end{array}\right], \operatorname{det}(j)=-j_{41} j_{53} j_{62}+j_{42} j_{53} j_{61}\right]} \\
{[\lambda=0, \eta=1, \nu=0, \mu=1, \varepsilon=0]}
\end{array}\right]
\end{aligned}
$$




$$
\begin{aligned}
& \begin{array}{c}
{\left[j=\left[\begin{array}{ccc}
j_{41} & j_{42} & 0 \\
j_{51} & j_{52} & 0 \\
j_{61} & 0 & j_{63}
\end{array}\right], \operatorname{det}(j)=j_{41} j_{52} j_{63}-j_{42} j_{51} j_{63}\right]} \\
{[\lambda=0, \eta=1, \nu=1, \mu=0, \varepsilon=0]}
\end{array} \\
& \begin{array}{c}
{\left[j=\left[\begin{array}{ccc}
j_{41} & 0 & j_{43} \\
j_{51} & j_{52} & 0 \\
j_{61} & j_{62} & 0
\end{array}\right], \operatorname{det}(j)=j_{43} j_{51} j_{62}-j_{43} j_{52} j_{61}\right]} \\
{[\lambda=0, \eta=0, \nu=1, \mu=1, \varepsilon=0]}
\end{array} \\
& \left.\begin{array}{c}
{\left[j=\left[\begin{array}{ccc}
j_{41} & j_{42} & 0 \\
j_{51} & 0 & 0 \\
j_{61} & 0 & j_{63}
\end{array}\right], \operatorname{det}(j)=-j_{42} j_{51} j_{63}\right]} \\
{[\lambda \neq 0, \lambda=\mu, \eta=1, \nu=\nu, \mu=\mu]}
\end{array}\right] \\
& \left.\begin{array}{c}
{\left[j=\left[\begin{array}{ccc}
j_{41} & j_{42} & 0 \\
j_{51} & 0 & j_{53} \\
j_{61} & 0 & 0
\end{array}\right], \operatorname{det}(j)=j_{42} j_{53} j_{61}\right]} \\
{[\lambda \neq 0, \lambda=\nu, \eta=1, \nu=\nu, \mu=\mu]}
\end{array}\right] \\
& \left.\begin{array}{c}
{\left[j=\left[\begin{array}{ccc}
j_{41} & 0 & j_{43} \\
j_{51} & 0 & 0 \\
j_{61} & j_{62} & 0
\end{array}\right], \operatorname{det}(j)=j_{43} j_{51} j_{62}\right]} \\
{[\lambda \neq 0, \lambda=\lambda, \eta=\lambda, \nu=\nu, \mu=1]}
\end{array}\right] \\
& \left.\begin{array}{c}
{\left[j=\left[\begin{array}{ccc}
j_{41} & 0 & j_{43} \\
j_{51} & j_{52} & 0 \\
j_{61} & 0 & 0
\end{array}\right], \operatorname{det}(j)=-j_{43} j_{52} j_{61}\right]} \\
{[\lambda \neq 0, \lambda=\lambda, \eta=\lambda, \nu=1, \mu=\mu]}
\end{array}\right] \\
& \left.\begin{array}{c}
{\left[j=\left[\begin{array}{ccc}
j_{41} & 0 & 0 \\
j_{51} & j_{52} & 0 \\
j_{61} & 0 & j_{63}
\end{array}\right], \operatorname{det}(j)=j_{41} j_{52} j_{63}\right]} \\
{[\lambda \neq 0, \lambda=\mu, \eta=\eta, \nu=1, \mu=\mu]}
\end{array}\right] \\
& \left.\begin{array}{c}
{\left[j=\left[\begin{array}{ccc}
j_{41} & 0 & 0 \\
j_{51} & 0 & j_{53} \\
j_{61} & j_{62} & 0
\end{array}\right], \operatorname{det}(j)=-j_{41} j_{53} j_{62}\right]} \\
{[\lambda \neq 0, \lambda=\nu, \eta=\eta, \nu=\nu, \mu=1]}
\end{array}\right] \\
& \begin{array}{c}
{\left[j=\left[\begin{array}{ccc}
j_{41} & j_{42} & 0 \\
j_{51} & 0 & j_{53} \\
j_{61} & 0 & j_{63}
\end{array}\right], \operatorname{det}(j)=-j_{42} j_{51} j_{63}+j_{42} j_{53} j_{61}\right]} \\
{[\lambda \neq 0, \lambda=\nu, \eta=1, \nu=\nu, \mu=\nu]}
\end{array}
\end{aligned}
$$


R. CAMPOAMOR STURSBERG, I. E. CARDOSO, AND G. P. OVANDO

$$
\begin{aligned}
& \left.\begin{array}{c}
{\left[j=\left[\begin{array}{ccc}
j_{41} & j_{42} & 0 \\
j_{51} & j_{52} & 0 \\
j_{61} & 0 & j_{63}
\end{array}\right], \operatorname{det}(j)=j_{41} j_{52} j_{63}-j_{42} j_{51} j_{63}\right]} \\
{[\lambda \neq 0, \lambda=\mu, \eta=1, \nu=1, \mu=\mu]}
\end{array}\right] \\
& \left.\begin{array}{c}
{\left[j=\left[\begin{array}{ccc}
j_{41} & j_{42} & 0 \\
j_{51} & 0 & j_{53} \\
j_{61} & j_{62} & 0
\end{array}\right], \operatorname{det}(j)=-j_{41} j_{53} j_{62}+j_{42} j_{53} j_{61}\right]} \\
{[\lambda \neq 0, \lambda=\nu, \eta=1, \nu=\nu, \mu=1]}
\end{array}\right] \\
& \left.\begin{array}{c}
{\left[j=\left[\begin{array}{ccc}
j_{41} & 0 & j_{43} \\
j_{51} & j_{52} & 0 \\
j_{61} & 0 & j_{63}
\end{array}\right], \operatorname{det}(j)=j_{41} j_{52} j_{63}-j_{43} j_{52} j_{61}\right]} \\
{[\lambda \neq 0, \lambda=\mu, \eta=\mu, \nu=1, \mu=\mu]}
\end{array}\right] \\
& \begin{array}{c}
{\left[j=\left[\begin{array}{ccc}
j_{41} & 0 & j_{43} \\
j_{51} & 0 & j_{53} \\
j_{61} & j_{62} & 0
\end{array}\right], \operatorname{det}(j)=-j_{41} j_{53} j_{62}+j_{43} j_{51} j_{62}\right]} \\
{[\lambda \neq 0, \lambda=\nu, \eta=\nu, \nu=\nu, \mu=1]}
\end{array} \\
& \begin{array}{c}
{\left[j=\left[\begin{array}{ccc}
j_{41} & 0 & j_{43} \\
j_{51} & j_{52} & 0 \\
j_{61} & j_{62} & 0
\end{array}\right], \operatorname{det}(j)=j_{43} j_{51} j_{62}-j_{43} j_{52} j_{61}\right]} \\
{[\lambda \neq 0, \lambda=\lambda, \eta=\lambda, \nu=1, \mu=1]}
\end{array} \\
& \begin{array}{c}
{\left[j=\left[\begin{array}{ccc}
j_{41} & j_{42} & j_{43} \\
j_{51} & 0 & 0 \\
j_{61} & j_{62} & j_{63}
\end{array}\right], \operatorname{det}(j)=-j_{51}\left(j_{42} j_{63}-j_{43} j_{62}\right)\right]} \\
{[\lambda \neq 0, \lambda=1, \eta=1, \nu=\nu, \mu=1]}
\end{array} \\
& \begin{array}{c}
{\left[j=\left[\begin{array}{ccc}
j_{41} & j_{42} & j_{43} \\
j_{51} & j_{52} & j_{53} \\
j_{61} & 0 & 0
\end{array}\right], \operatorname{det}(j)=j_{61}\left(j_{42} j_{53}-j_{43} j_{52}\right)\right]} \\
{[\lambda \neq 0, \lambda=1, \eta=1, \nu=1, \mu=\mu]}
\end{array} \\
& \begin{array}{c}
{\left[j=\left[\begin{array}{ccc}
j_{41} & 0 & 0 \\
j_{51} & j_{52} & j_{53} \\
j_{61} & j_{62} & j_{63}
\end{array}\right], \operatorname{det}(j)=j_{41}\left(j_{52} j_{63}-j_{53} j_{62}\right)\right]} \\
{[\lambda \neq 0, \lambda=1, \eta=\eta, \nu=1, \mu=1]}
\end{array}
\end{aligned}
$$$$
\begin{gathered}
{\left[j=\left[\begin{array}{ccc}
j_{41} & j_{42} & j_{43} \\
j_{51} & j_{52} & j_{53} \\
j_{61} & j_{62} & j_{63}
\end{array}\right], \operatorname{det}(j)=j_{41} j_{52} j_{63}-j_{41} j_{53} j_{62}-j_{42} j_{51} j_{63}+j_{42} j_{53} j_{61}+j_{43} j_{51} j_{62}-j_{43} j_{52} j_{61}\right]} \\
{[\lambda \neq 0, \lambda=1, \eta=1, \nu=1, \mu=1]}
\end{gathered}
$$ 
(b) For a type (ii) representation: there are 26 solutions.

$$
\begin{aligned}
& \left.\begin{array}{c}
{\left[j=\left[\begin{array}{ccc}
j_{41} & 0 & 0 \\
j_{51} & -i j_{62} & i j_{63} \\
j_{61} & j_{62} & j_{63}
\end{array}\right], \operatorname{det}(j)=-2 i j_{41} j_{62} j_{63}\right]} \\
{[\lambda=0, \eta=\eta, \nu=1 / 2, \mu=1 / 2 i, \varepsilon=0]}
\end{array}\right] \\
& \left.\begin{array}{c}
{\left[j=\left[\begin{array}{ccc}
j_{41} & 0 & 0 \\
j_{51} & i j_{62} & -i j_{63} \\
j_{61} & j_{62} & j_{63}
\end{array}\right], \operatorname{det}(j)=2 i j_{41} j_{63} j_{62}\right]} \\
{[\lambda=0, \eta=\eta, \nu=1 / 2, \mu=-1 / 2 i, \varepsilon=0]}
\end{array}\right] \\
& \begin{array}{c}
{\left[j=\left[\begin{array}{ccc}
j_{41} & j_{42} & 0 \\
j_{51} & -i j_{62} & i j_{63} \\
j_{61} & j_{62} & j_{63}
\end{array}\right], \operatorname{det}(j)=-2 i j_{41} j_{62} j_{63}-j_{51} j_{42} j_{63}+i j_{61} j_{42} j_{63}\right]} \\
{[\lambda=0, \eta=1, \nu=1 / 2, \mu=1 / 2 i, \varepsilon=0]}
\end{array} \\
& \begin{array}{c}
{\left[j=\left[\begin{array}{ccc}
j_{41} & j_{42} & 0 \\
j_{51} & i j_{62} & -i j_{63} \\
j_{61} & j_{62} & j_{63}
\end{array}\right], \operatorname{det}(j)=2 i j_{41} j_{63} j_{62}-j_{51} j_{42} j_{63}-i j_{61} j_{42} j_{63}\right]} \\
{[\lambda=0, \eta=1, \nu=1 / 2, \mu=-1 / 2 i, \varepsilon=0]}
\end{array} \\
& {\left[\begin{array}{c}
{\left[j=\left[\begin{array}{ccc}
j_{41} & j_{42} & 0 \\
j_{51} & 0 & j_{53} \\
j_{61} & 0 & j_{63}
\end{array}\right], \operatorname{det}(j)=-j_{51} j_{42} j_{63}+j_{61} j_{42} j_{53}\right]} \\
{[\lambda=0, \eta=1, \nu=0, \mu=0, \varepsilon=0]}
\end{array}\right]} \\
& \left.\begin{array}{c}
{\left[j=\left[\begin{array}{ccc}
j_{41} & 0 & j_{43} \\
j_{51} & -i j_{62} & i j_{63} \\
j_{61} & j_{62} & j_{63}
\end{array}\right], \operatorname{det}(j)=-2 i j_{41} j_{62} j_{63}+j_{43} j_{51} j_{62}+i j_{61} j_{43} j_{62}\right]} \\
{[\lambda=0, \eta=0, \nu=1 / 2, \mu=1 / 2 i, \varepsilon=0]}
\end{array}\right] \\
& \left.\begin{array}{c}
{\left[j=\left[\begin{array}{ccc}
j_{41} & 0 & j_{43} \\
j_{51} & i j_{62} & -i j_{63} \\
j_{61} & j_{62} & j_{63}
\end{array}\right], \operatorname{det}(j)=2 i j_{41} j_{63} j_{62}+j_{43} j_{51} j_{62}-i j_{61} j_{43} j_{62}\right]} \\
{[\lambda=0, \eta=0, \nu=1 / 2, \mu=-1 / 2 i, \varepsilon=0]}
\end{array}\right] \\
& {\left[\begin{array}{c}
{\left[j=\left[\begin{array}{ccc}
j_{41} & 0 & j_{43} \\
j_{51} & j_{52} & 0 \\
j_{61} & j_{62} & 0
\end{array}\right], \operatorname{det}(j)=j_{43} j_{51} j_{62}-j_{43} j_{52} j_{61}\right]} \\
{[\lambda=0, \eta=0, \nu=1, \mu=0, \varepsilon=0]}
\end{array}\right]} \\
& \begin{array}{c}
{\left[j=\left[\begin{array}{ccc}
j_{41} & j_{42} & j_{43} \\
j_{51} & j_{52} & j_{53} \\
j_{61} & j_{62} & j_{63}
\end{array}\right], \operatorname{det}(j)=j_{41} j_{52} j_{63}-j_{41} j_{53} j_{62}-j_{42} j_{51} j_{63}+j_{42} j_{53} j_{61}+j_{43} j_{51} j_{62}-j_{43} j_{52} j_{61}\right]} \\
{[\lambda \neq 0, \lambda=1, \eta=1, \nu=1, \mu=0]}
\end{array}
\end{aligned}
$$


R. CAMPOAMOR STURSBERG, I. E. CARDOSO, AND G. P. OVANDO

$$
\begin{aligned}
& \left.\begin{array}{c}
{\left[j=\left[\begin{array}{ccc}
j_{41} & 0 & 0 \\
j_{51} & j_{52} & j_{53} \\
j_{61} & j_{62} & j_{63}
\end{array}\right], \operatorname{det}(j)=j_{41}\left(j_{52} j_{63}-j_{53} j_{62}\right)\right]} \\
{[\lambda \neq 0, \lambda=1, \eta=\eta, \nu=1, \mu=0]}
\end{array}\right] \\
& \left.\begin{array}{c}
{\left[j=\left[\begin{array}{ccc}
j_{41} & 0 & 0 \\
j_{51} & i j_{62} & -i j_{63} \\
j_{61} & j_{62} & j_{63}
\end{array}\right], \operatorname{det}(j)=2 i j_{41} j_{62} j_{63}\right]} \\
{[\lambda \neq 0, \lambda=2 \nu-1, \eta=\eta, \nu=\nu, \mu=i(\nu-1)]}
\end{array}\right] \\
& \left.\begin{array}{c}
{\left[j=\left[\begin{array}{ccc}
j_{41} & 0 & 0 \\
j_{51} & -i j_{62} & i j_{63} \\
j_{61} & j_{62} & j_{63}
\end{array}\right], \operatorname{det}(j)=-2 i j_{41} j_{63} j_{62}\right]} \\
{[\lambda \neq 0, \lambda=2 \nu-1, \eta=\eta, \nu=\nu, \mu=-i(\nu-1)]}
\end{array}\right] \\
& \left.\begin{array}{c}
{\left[j=\left[\begin{array}{ccc}
j_{41} & j_{42} & 0 \\
j_{51} & 0 & -i j_{63} \\
j_{61} & 0 & j_{63}
\end{array}\right], \operatorname{det}(j)=-j_{42} j_{51} j_{63}-i j_{61} j_{42} j_{63}\right]} \\
{[\lambda \neq 0, \lambda=\lambda, \eta=1, \nu=\nu, \mu=i(\lambda-\nu)]}
\end{array}\right] \\
& \begin{array}{c}
{\left[j=\left[\begin{array}{ccc}
j_{41} & j_{42} & 0 \\
j_{51} & 0 & i j_{63} \\
j_{61} & 0 & j_{63}
\end{array}\right], \operatorname{det}(j)=-j_{42} j_{51} j_{63}+i j_{61} j_{42} j_{63}\right]} \\
{[\lambda \neq 0, \lambda=\lambda, \eta=1, \nu=\nu, \mu=-i(\lambda-\nu)]}
\end{array} \\
& \begin{array}{c}
{\left[j=\left[\begin{array}{ccc}
j_{41} & j_{42} & 0 \\
j_{51} & 0 & j_{53} \\
j_{61} & 0 & j_{63}
\end{array}\right], \operatorname{det}(j)=-j_{42} j_{51} j_{63}+j_{42} j_{53} j_{61}\right]} \\
{[\lambda \neq 0, \lambda=\nu, \eta=1, \nu=\nu, \mu=0]}
\end{array} \\
& \begin{array}{c}
{\left[j=\left[\begin{array}{ccc}
j_{41} & j_{42} & 0 \\
j_{51} & i j_{62} & i j_{63} \\
j_{61} & j_{62} & j_{63}
\end{array}\right], \operatorname{det}(j)=-j_{42} j_{51} j_{63}+i j_{61} j_{42} j_{63}\right]} \\
{[\lambda \neq 0, \lambda=1, \eta=1, \nu=\nu, \mu=i(\nu-1)]}
\end{array} \\
& \left.\begin{array}{c}
{\left[j=\left[\begin{array}{ccc}
j_{41} & j_{42} & 0 \\
j_{51} & -i j_{62} & -i j_{63} \\
j_{61} & j_{62} & j_{63}
\end{array}\right], \operatorname{det}(j)=-j_{42} j_{51} j_{63}-i j_{61} j_{42} j_{63}\right]} \\
{[\lambda \neq 0, \lambda=1, \eta=1, \nu=\nu, \mu=-i(\nu-1)]}
\end{array}\right] \\
& \left.\begin{array}{c}
{\left[j=\left[\begin{array}{ccc}
j_{41} & j_{42} & 0 \\
j_{51} & i j_{62} & -i j_{63} \\
j_{61} & j_{62} & j_{63}
\end{array}\right], \operatorname{det}(j)=2 i j_{41} j_{62} j_{63}-j_{42} j_{51} j_{63}-i j_{61} j_{42} j_{63}\right]} \\
{[\lambda \neq 0, \lambda=2 \nu-1, \eta=1, \nu=\nu, \mu=i(\nu-1)]}
\end{array}\right]
\end{aligned}
$$




$$
\begin{aligned}
& \left.\begin{array}{c}
{\left[j=\left[\begin{array}{ccc}
j_{41} & j_{42} & 0 \\
j_{51} & -i j_{62} & i j_{63} \\
j_{61} & j_{62} & j_{63}
\end{array}\right], \operatorname{det}(j)=-2 i j_{41} j_{63} j_{62}-j_{42} j_{51} j_{63}+i j_{61} j_{42} j_{63}\right]} \\
{[\lambda \neq 0, \lambda=2 \nu-1, \eta=1, \nu=\nu, \mu=-i(\nu-1)]}
\end{array}\right] \\
& \left.\begin{array}{c}
{\left[j=\left[\begin{array}{ccc}
j_{41} & 0 & j_{43} \\
j_{51} & i j_{62} & 0 \\
j_{61} & j_{62} & 0
\end{array}\right], \operatorname{det}(j)=j_{43} j_{51} j_{62}-i j_{61} j_{43} j_{62}\right]} \\
{[\lambda \neq 0, \lambda=\lambda, \eta=\lambda, \nu=\nu, \mu=i(\nu-1)]}
\end{array}\right] \\
& \left.\begin{array}{c}
{\left[j=\left[\begin{array}{ccc}
j_{41} & 0 & j_{43} \\
j_{51} & -i j_{62} & 0 \\
j_{61} & j_{62} & 0
\end{array}\right], \operatorname{det}(j)=j_{43} j_{51} j_{62}+i j_{61} j_{43} j_{62}\right]} \\
{[\lambda \neq 0, \lambda=\lambda, \eta=\lambda, \nu=\nu, \mu=-i(\nu-1)]}
\end{array}\right] \\
& \left.\begin{array}{c}
{\left[j=\left[\begin{array}{ccc}
j_{41} & 0 & j_{43} \\
j_{51} & j_{52} & 0 \\
j_{61} & j_{62} & 0
\end{array}\right], \operatorname{det}(j)=j_{43} j_{51} j_{62}-j_{43} j_{52} j_{61}\right]} \\
{[\lambda \neq 0, \lambda=\lambda, \eta=\lambda, \nu=1, \mu=0]}
\end{array}\right] \\
& \left.\begin{array}{c}
{\left[j=\left[\begin{array}{ccc}
j_{41} & 0 & j_{43} \\
j_{51} & i j_{62} & i j_{63} \\
j_{61} & j_{62} & j_{63}
\end{array}\right], \operatorname{det}(j)=j_{43} j_{51} j_{62}-i j_{61} j_{43} j_{62}\right]} \\
{[\lambda \neq 0, \lambda=1, \eta=1, \nu=\nu, \mu=i(\nu-1)]}
\end{array}\right] \\
& \left.\begin{array}{c}
{\left[j=\left[\begin{array}{ccc}
j_{41} & 0 & j_{43} \\
j_{51} & -i j_{62} & -i j_{63} \\
j_{61} & j_{62} & j_{63}
\end{array}\right], \operatorname{det}(j)=j_{43} j_{51} j_{62}+i j_{61} j_{43} j_{62}\right]} \\
{[\lambda \neq 0, \lambda=1, \eta=1, \nu=\nu, \mu=-i(\nu-1)]}
\end{array}\right] \\
& {\left[\begin{array}{c}
{\left[j=\left[\begin{array}{ccc}
j_{41} & 0 & j_{43} \\
j_{51} & i j_{62} & -i j_{63} \\
j_{61} & j_{62} & j_{63}
\end{array}\right], \operatorname{det}(j)=2 i j_{41} j_{62} j_{63}+j_{43} j_{51} j_{62}-i j_{61} j_{43} j_{62}\right]} \\
{[\lambda \neq 0, \lambda=2 \nu-1, \eta=2 \nu-1, \nu=\nu, \mu=i(\nu-1)]}
\end{array}\right]} \\
& \left.\begin{array}{c}
{\left[j=\left[\begin{array}{ccc}
j_{41} & 0 & j_{43} \\
j_{51} & -i j_{62} & i j_{63} \\
j_{61} & j_{62} & j_{63}
\end{array}\right], \operatorname{det}(j)=-2 i j_{41} j_{63} j_{62}+j_{43} j_{51} j_{62}+i j_{61} j_{43} j_{62}\right]} \\
{[\lambda \neq 0, \lambda=2 \nu-1, \eta=2 \nu-1, \nu=\nu, \mu=-i(\nu-1)]}
\end{array}\right]
\end{aligned}
$$

(c) For a type (iii) there are 5 solutions.

$$
\begin{gathered}
{\left[j=\left[\begin{array}{ccc}
j_{41} & j_{42} & 0 \\
j_{51} & 0 & j_{53} \\
j_{61} & 0 & 0
\end{array}\right], \operatorname{det}(j)=j_{42} j_{53} j_{61}\right]} \\
{[\lambda=0, \eta=1, \nu=0, \varepsilon=0]}
\end{gathered}
$$




$$
\left.\begin{array}{c}
{\left[j=\left[\begin{array}{ccc}
j_{41} & 0 & j_{43} \\
j_{51} & j_{52} & 0 \\
j_{61} & 0 & 0
\end{array}\right], \operatorname{det}(j)=-j_{43} j_{52} j_{61}\right]} \\
{[\lambda=0, \eta=0, \nu=1, \varepsilon=0]}
\end{array}\right]
$$

(d) For a type (iii) there are no solutions.

(4) CASE IV: $\mathfrak{k}=\mathfrak{r}_{3, \delta}$.

(a) For a type (i) representation: there are 16 solutions.

$$
\begin{aligned}
& \left.\begin{array}{c}
{\left[j=\left[\begin{array}{ccc}
j_{41} & 0 & 0 \\
j_{51} & j_{52} & j_{53} \\
j_{61} & j_{62} & j_{63}
\end{array}\right], \operatorname{det}(j)=j_{41}\left(j_{52} j_{63}-j_{53} j_{62}\right)\right]} \\
{[\delta=0, \eta=\eta, \nu=0, \mu=1]}
\end{array}\right] \\
& \left.\begin{array}{c}
{\left[j=\left[\begin{array}{ccc}
j_{41} & j_{42} & j_{43} \\
j_{51} & j_{52} & j_{53} \\
j_{61} & j_{62} & j_{63}
\end{array}\right], \operatorname{det}(j)=j_{41} j_{52} j_{63}-j_{41} j_{53} j_{62}-j_{42} j_{51} j_{63}+j_{42} j_{53} j_{61}+j_{43} j_{51} j_{62}-j_{43} j_{52} j_{61}\right]} \\
{[\delta=0, \eta=1, \nu=0, \mu=1]}
\end{array}\right] \\
& \left.\begin{array}{c}
{\left[j=\left[\begin{array}{ccc}
j_{41} & j_{42} & j_{43} \\
j_{51} & 0 & 0 \\
j_{61} & j_{62} & j_{63}
\end{array}\right], \operatorname{det}(j)=-j_{51}\left(j_{42} j_{63}-j_{43} j_{62}\right)\right]} \\
{[\delta=0, \eta=1, \nu=0, \mu=1]}
\end{array}\right] \\
& \begin{array}{c}
{\left[j=\left[\begin{array}{ccc}
j_{41} & j_{42} & j_{43} \\
j_{51} & j_{52} & j_{53} \\
j_{61} & 0 & 0
\end{array}\right], \operatorname{det}(j)=j_{61}\left(j_{42} j_{53}-j_{43} j_{52}\right)\right]} \\
{[\delta=0, \eta=1, \nu=0, \mu=\mu]}
\end{array} \\
& {\left[\begin{array}{c}
{\left[j=\left[\begin{array}{ccc}
j_{41} & -i j_{43} & j_{43} \\
j_{51} & i j_{53} & j_{53} \\
j_{61} & 0 & 0
\end{array}\right], \operatorname{det}(j)=-2 i j_{61} j_{43} j_{53}\right]} \\
{[\delta=-i(\eta-1), \eta=\eta, \nu=-i(\eta-1), \mu=\mu]}
\end{array}\right]}
\end{aligned}
$$


ADDENDUM TO "EXTENDING INVARIANT COMPLEX STRUCTURES"

$$
\begin{aligned}
& {\left[\begin{array}{c}
{\left[j=\left[\begin{array}{ccc}
j_{41} & i j_{43} & j_{43} \\
j_{51} & -i j_{53} & j_{53} \\
j_{61} & 0 & 0
\end{array}\right], \operatorname{det}(j)=2 i j_{61} j_{43} j_{53}\right]} \\
{[\delta=i(\eta-1), \eta=\eta, \nu=i(\eta-1), \mu=\mu]}
\end{array}\right]} \\
& \left.\begin{array}{c}
{\left[j=\left[\begin{array}{ccc}
j_{41} & 0 & 0 \\
j_{51} & -i j_{53} & j_{53} \\
j_{61} & i j_{63} & j_{63}
\end{array}\right], \operatorname{det}(j)=-2 i j_{41} j_{53} j_{63}\right]} \\
{[\delta=-i(\nu-1), \eta=\eta, \nu=-i(\nu-1), \mu=2-\nu]}
\end{array}\right] \\
& \left.\begin{array}{c}
{\left[j=\left[\begin{array}{ccc}
j_{41} & 0 & 0 \\
j_{51} & i j_{53} & j_{53} \\
j_{61} & -i j_{63} & j_{63}
\end{array}\right], \operatorname{det}(j)=2 i j_{41} j_{53} j_{63}\right]} \\
{[\delta=i(\nu-1), \eta=\eta, \nu=i(\nu-1), \mu=2-\nu]}
\end{array}\right] \\
& {\left[\begin{array}{c}
{\left[j=\left[\begin{array}{ccc}
j_{41} & -i j_{43} & j_{43} \\
j_{51} & 0 & 0 \\
j_{61} & i j_{63} & j_{63}
\end{array}\right], \operatorname{det}(j)=2 i j_{51} j_{43} j_{63}\right]} \\
{[\delta=-i(\eta-1), \eta=\eta, \nu=-i(\eta-1), \mu=-\eta+2]}
\end{array}\right]} \\
& \left.\begin{array}{c}
{\left[j=\left[\begin{array}{ccc}
j_{41} & i j_{43} & j_{43} \\
j_{51} & 0 & 0 \\
j_{61} & -i j_{63} & j_{63}
\end{array}\right], \operatorname{det}(j)=-2 i j_{51} j_{43} j_{63}\right]} \\
{[\delta=i(\eta-1), \eta=\eta, \nu=i(\eta-1), \mu=-\eta+2]}
\end{array}\right] \\
& \left.\begin{array}{c}
{\left[j=\left[\begin{array}{ccc}
j_{41} & -i j_{43} & j_{43} \\
j_{51} & -i j_{53} & j_{53} \\
j_{61} & i j_{63} & j_{63}
\end{array}\right], \operatorname{det}(j)=-2 i j_{41} j_{53} j_{63}+2 i j_{51} j_{43} j_{63}\right]} \\
{[\delta=-i(\nu-1), \eta=\nu, \nu=-i(\nu-1), \mu=2-\nu]}
\end{array}\right] \\
& \begin{array}{c}
{\left[j=\left[\begin{array}{ccc}
j_{41} & i j_{43} & j_{43} \\
j_{51} & i j_{53} & j_{53} \\
j_{61} & -i j_{63} & j_{63}
\end{array}\right], \operatorname{det}(j)=2 i j_{41} j_{53} j_{63}-2 i j_{51} j_{43} j_{63}\right]} \\
{[\delta=i(\nu-1), \eta=\nu, \nu=i(\nu-1), \mu=2-\nu]}
\end{array} \\
& \begin{array}{c}
{\left[j=\left[\begin{array}{ccc}
j_{41} & -i j_{43} & j_{43} \\
j_{51} & i j_{53} & j_{53} \\
j_{61} & i j_{63} & j_{63}
\end{array}\right], \operatorname{det}(j)=2 i j_{51} j_{43} j_{63}-2 i j_{61} j_{43} j_{53}\right]} \\
{[\delta=i(\nu-1), \eta=2-\nu, \nu=i(\nu-1), \mu=\nu]}
\end{array} \\
& \left.\begin{array}{c}
{\left[j=\left[\begin{array}{ccc}
j_{41} & i j_{43} & j_{43} \\
j_{51} & -i j_{53} & j_{53} \\
j_{61} & i j_{63} & j_{63}
\end{array}\right], \operatorname{det}(j)=-2 i j_{41} j_{53} j_{63}+2 i j_{61} j_{43} j_{53}\right]} \\
{[\delta=-i(\nu-1), \eta=2-\nu, \nu=-i(\nu-1), \mu=2-\nu]}
\end{array}\right]
\end{aligned}
$$




$$
\begin{aligned}
& \left.\begin{array}{c}
{\left[j=\left[\begin{array}{ccc}
j_{41} & -i j_{43} & j_{43} \\
j_{51} & i j_{53} & j_{53} \\
j_{61} & -i j_{63} & j_{63}
\end{array}\right], \operatorname{det}(j)=2 i j_{41} j_{53} j_{63}-2 i j_{61} j_{43} j_{53}\right]} \\
{[\delta=i(\nu-1), \eta=2-\nu, \nu=i(\nu-1), \mu=2-\nu]}
\end{array}\right] \\
& \begin{array}{c}
{\left[j=\left[\begin{array}{ccc}
j_{41} & i j_{43} & j_{43} \\
j_{51} & -i j_{53} & j_{53} \\
j_{61} & -i j_{63} & j_{63}
\end{array}\right], \operatorname{det}(j)=-2 i j_{51} j_{43} j_{63}+2 i j_{61} j_{43} j_{53}\right]} \\
{[\delta=-i(\nu-1), \eta=2-\nu, \nu=-i(\nu-1), \mu=\nu]}
\end{array}
\end{aligned}
$$

(b) For a type (ii) representation: there are 16 solutions.

$$
\begin{aligned}
& \left.\begin{array}{c}
{\left[j=\left[\begin{array}{ccc}
j_{41} & i j_{43} & j_{43} \\
j_{51} & j_{63} & j_{53} \\
j_{61} & -j_{53} & j_{63}
\end{array}\right], \operatorname{det}(j)=-i j_{43} j_{51} j_{63}+i j_{43} j_{53} j_{61}+j_{41} j_{53}{ }^{2}+j_{41} j_{63}{ }^{2}-j_{43} j_{51} j_{53}-j_{43} j_{61} j_{63}\right]} \\
{[\delta=\mu, \eta=-i \mu+1, \nu=1, \mu=\mu]}
\end{array}\right] \\
& \begin{array}{c}
{\left[j=\left[\begin{array}{ccc}
j_{41} & -i j_{43} & j_{43} \\
j_{51} & j_{63} & j_{53} \\
j_{61} & -j_{53} & j_{63}
\end{array}\right], \operatorname{det}(j)=i j_{43} j_{51} j_{63}-i j_{43} j_{53} j_{61}+j_{41} j_{53}{ }^{2}+j_{41} j_{63}{ }^{2}-j_{43} j_{51} j_{53}-j_{43} j_{61} j_{63}\right]} \\
{[\delta=\mu, \eta=i \mu+1, \nu=1, \mu=\mu]}
\end{array} \\
& \left.\begin{array}{c}
{\left[j=\left[\begin{array}{ccc}
j_{41} & i j_{43} & j_{43} \\
j_{51} & -j_{63} & j_{53} \\
j_{61} & j_{53} & j_{63}
\end{array}\right], \operatorname{det}(j)=-i j_{43} j_{51} j_{63}+i j_{43} j_{53} j_{61}-j_{41} j_{53}{ }^{2}-j_{41} j_{63}{ }^{2}+j_{43} j_{51} j_{53}+j_{43} j_{61} j_{63}\right]} \\
{[\delta=-\mu, \eta=i \mu+1, \nu=1, \mu=\mu]}
\end{array}\right] \\
& \begin{array}{c}
{\left[j=\left[\begin{array}{ccc}
j_{41} & -i j_{43} & j_{43} \\
j_{51} & -j_{63} & j_{53} \\
j_{61} & j_{53} & j_{63}
\end{array}\right], \operatorname{det}(j)=i j_{43} j_{51} j_{63}-i j_{43} j_{53} j_{61}-j_{41} j_{53}{ }^{2}-j_{41} j_{63}{ }^{2}+j_{43} j_{51} j_{53}+j_{43} j_{61} j_{63}\right]} \\
{[\delta=-\mu, \eta=-i \mu+1, \nu=1, \mu=\mu]}
\end{array} \\
& \left.\begin{array}{c}
{\left[j=\left[\begin{array}{ccc}
j_{41} & j_{42} & j_{43} \\
j_{51} & i j_{62} & i j_{63} \\
j_{61} & j_{62} & j_{63}
\end{array}\right]\right.} \\
{[\delta=0, \eta=1, \nu=\nu, \mu=i(\nu-1)]}
\end{array}\right] \\
& \left.\begin{array}{c}
{\left[j=\left[\begin{array}{ccc}
j_{41} & j_{42} & j_{43} \\
j_{51} & -i j_{62} & -i j_{63} \\
j_{61} & j_{62} & j_{63}
\end{array}\right], \operatorname{det}(j)=-i j_{42} j_{61} j_{63}+i j_{43} j_{61} j_{62}-j_{42} j_{51} j_{63}+j_{43} j_{51} j_{62}\right]} \\
{[\delta=0, \eta=1, \nu=\nu, \mu=-i(\nu-1)]}
\end{array}\right]
\end{aligned}
$$

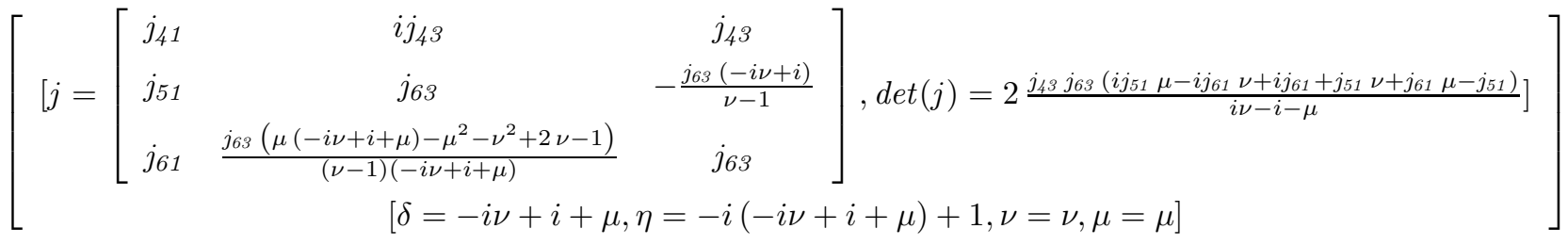


ADDENDUM TO "EXTENDING INVARIANT COMPLEX STRUCTURES"

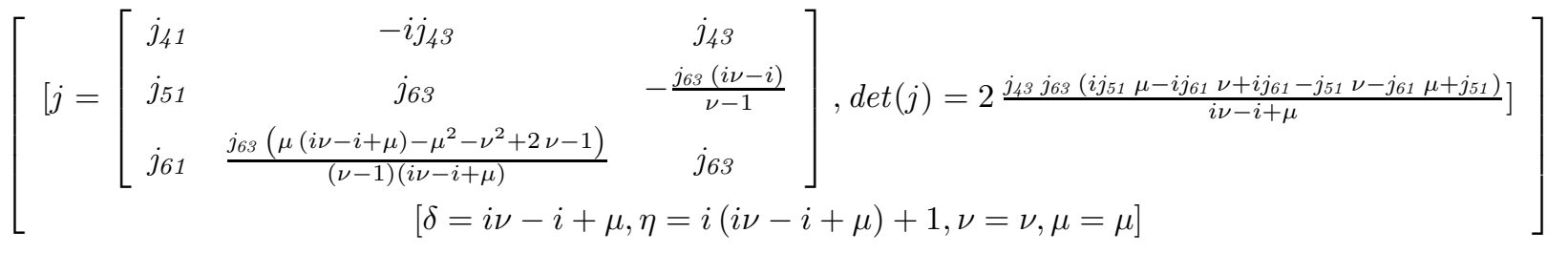

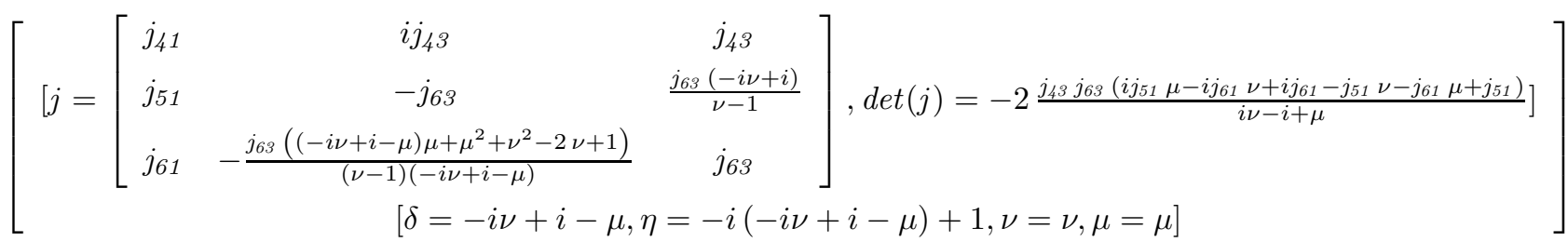

$$
\begin{aligned}
& {\left[\begin{array}{ccc}
{\left[j=\left[\begin{array}{ccc}
j_{41} & -i j_{43} & j_{43} \\
j_{51} & -j_{63} & \frac{j_{63}(i \nu-i)}{\nu-1} \\
j_{61} & -\frac{j_{63}\left((i \nu-i-\mu) \mu+\mu^{2}+\nu^{2}-2 \nu+1\right)}{(\nu-1)(i \nu-i-\mu)} & j_{63}
\end{array}\right], \operatorname{det}(j)=-2 \frac{j_{43} j_{63}\left(i j_{51} \mu-i j_{61} \nu+i j_{61}+j_{51} \nu+j_{61} \mu-j_{51}\right)}{i \nu-i-\mu}\right]} \\
{\left[\begin{array}{c}
{[\delta=i \nu-i-\mu, \eta} \\
{[}
\end{array}\right.}
\end{array}\right.} \\
& {\left[\begin{array}{c}
{\left[j=\left[\begin{array}{ccc}
j_{41} & 0 & 0 \\
j_{51} & j_{63} & -j_{62} \\
j_{61} & j_{62} & j_{63}
\end{array}\right], \operatorname{det}(j)=j_{41}\left(j_{62}{ }^{2}+j_{63}{ }^{2}\right)\right]} \\
{[\delta=\mu, \eta=\eta, \nu=1, \mu=\mu]}
\end{array}\right]} \\
& \begin{array}{c}
{\left[j=\left[\begin{array}{ccc}
j_{41} & 0 & 0 \\
j_{51} & -j_{63} & j_{62} \\
j_{61} & j_{62} & j_{63}
\end{array}\right], \operatorname{det}(j)=j_{41}\left(-j_{62}{ }^{2}-j_{63}{ }^{2}\right)\right]} \\
{[\delta=-\mu, \eta=\eta, \nu=1, \mu=\mu]}
\end{array} \\
& \begin{array}{c}
{\left[j=\left[\begin{array}{ccc}
j_{41} & 0 & 0 \\
j_{51} & j_{52} & j_{53} \\
j_{61} & j_{62} & j_{63}
\end{array}\right], \operatorname{det}(j)=j_{41}\left(j_{52} j_{63}-j_{53} j_{62}\right)\right]} \\
{[\delta=0, \eta=\eta, \nu=1, \mu=0]}
\end{array} \\
& \begin{array}{c}
{\left[j=\left[\begin{array}{ccc}
j_{41} & i j_{43} & j_{43} \\
j_{51} & -i j_{53} & j_{53} \\
j_{61} & -i j_{63} & j_{63}
\end{array}\right], \operatorname{det}(j)=-2 i j_{51} j_{43} j_{63}+2 i j_{61} j_{43} j_{53}\right]} \\
{[\delta=-i(\nu-1), \eta=-\nu+2, \nu=\nu, \mu=0]}
\end{array} \\
& \begin{array}{c}
{\left[j=\left[\begin{array}{ccc}
j_{41} & -i j_{43} & j_{43} \\
j_{51} & i j_{53} & j_{53} \\
j_{61} & i j_{63} & j_{63}
\end{array}\right], \operatorname{det}(j)=2 i j_{51} j_{43} j_{63}-2 i j_{61} j_{43} j_{53}\right]} \\
{[\delta=i(\nu-1), \eta=-\nu+2, \nu=\nu, \mu=0]}
\end{array} \\
& \begin{array}{c}
{\left[j=\left[\begin{array}{ccc}
j_{41} & j_{42} & j_{43} \\
j_{51} & j_{52} & j_{53} \\
j_{61} & j_{62} & j_{63}
\end{array}\right], \operatorname{det}(j)=j_{41} j_{52} j_{63}-j_{41} j_{53} j_{62}-j_{42} j_{51} j_{63}+j_{42} j_{53} j_{61}+j_{43} j_{51} j_{62}-j_{43} j_{52} j_{61}\right]} \\
{[\delta=0, \eta=1, \nu=1, \mu=0]}
\end{array}
\end{aligned}
$$


(c) For a type (iii) representation: there are 3 solutions.

$$
\left.\begin{array}{c}
{\left[j=\left[\begin{array}{ccc}
j_{41} & j_{42} & j_{43} \\
j_{51} & j_{52} & j_{53} \\
j_{61} & 0 & 0
\end{array}\right], \operatorname{det}(j)=j_{61}\left(j_{42} j_{53}-j_{43} j_{52}\right)\right]} \\
{[\delta=0, \eta=1, \nu=1]}
\end{array}\right]
$$

(d) For a type (iv) representation: there are no solutions.

This gives the results in Table 1 .

\subsection{Computations for $\mathfrak{h}=\mathfrak{h}_{1}$.}

(1) CASE I: $\mathfrak{k}=\mathfrak{h}_{1}$.

(a) For a type (i) representation: there are 2 solutions.

$$
\begin{aligned}
& {\left[\begin{array}{c}
{\left[j=\left[\begin{array}{ccc}
j_{41} & j_{42} & -\varepsilon \eta j_{41}-\varepsilon j_{51} \nu+\eta j_{42}+\nu j_{52} \\
j_{51} & j_{52} & \frac{\eta\left(\varepsilon \eta j_{41}+\varepsilon j_{51} \nu-\eta j_{42}-\nu j_{52}\right)}{\nu} \\
j_{61} & j_{62} & 0
\end{array}\right],\right.} \\
\left.\operatorname{det}(j)=-\frac{\left(\eta j_{41} j_{62}-j_{42} j_{61} \eta+j_{51} j_{62} \nu-j_{52} j_{61} \nu\right)\left(\varepsilon \eta j_{41}+\varepsilon j_{51} \nu-\eta j_{42}-\nu j_{52}\right)}{\nu}\right] \\
{\left[\eta=\eta, \nu=\nu, \mu=-\frac{\eta^{2}}{\nu}, \varepsilon=\varepsilon\right]}
\end{array}\right]} \\
& \left.\begin{array}{c}
{\left[j=\left[\begin{array}{ccc}
j_{41} & j_{42} & 0 \\
j_{51} & j_{52} & -\varepsilon j_{41} \mu+\mu j_{42} \\
j_{61} & j_{62} & 0
\end{array}\right]\right.} \\
{[\eta=0, \nu=0, \mu=\mu, \varepsilon=\varepsilon]} \\
{\left[\operatorname{det}(j)=\varepsilon j_{41}{ }^{2} j_{62} \mu-\varepsilon j_{41} j_{42} j_{61} \mu-j_{41} j_{42} j_{62} \mu+j_{42}{ }^{2} j_{61} \mu\right]}
\end{array}\right]
\end{aligned}
$$

(b) For a type (ii) representation: there are no solutions.

(2) CASE II: $\mathfrak{k}=\mathfrak{r}_{3}$.

(a) For a type (i) representation: there are no solutions.

(b) For a type (ii) representation: there are no solutions.

(3) CASE III: $\mathfrak{k}=\mathfrak{r}_{3, \lambda}$.

(a) For a type (i) representation: there are 6 solutions.

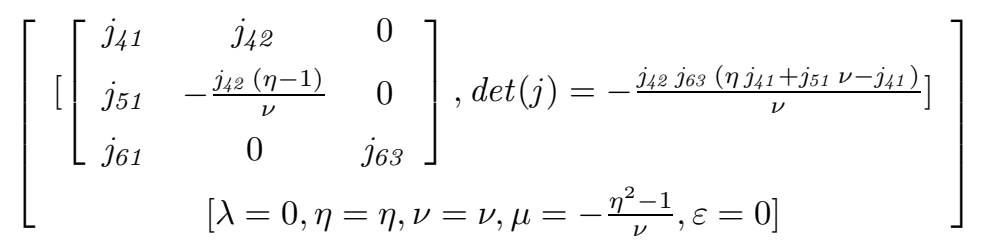




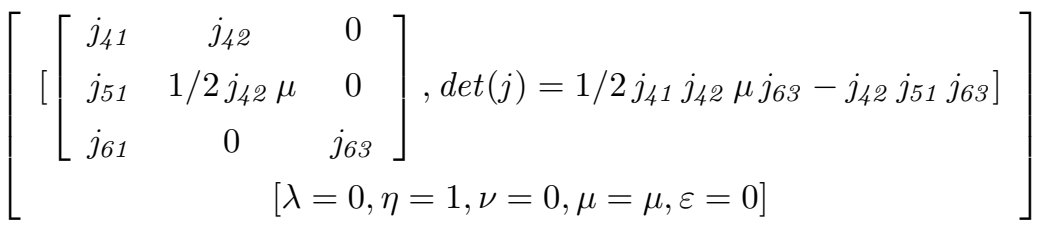

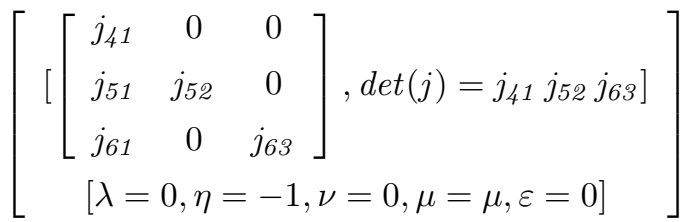

$$
\begin{aligned}
& \begin{array}{c}
{\left[j==\left[\begin{array}{ccc}
j_{41} & j_{42} & -1 / 2 j_{53} \nu \\
j_{51} & 0 & j_{53} \\
j_{61} & 0 & 0
\end{array}\right], \operatorname{det}(j)=j_{42} j_{53} j_{61}\right]} \\
{[\lambda \neq 0, \lambda=-1, \eta=1, \nu=\nu, \mu=0]}
\end{array} \\
& \left.\begin{array}{c}
{\left[j==\left[\begin{array}{ccc}
j_{41} & \frac{j_{52}\left(j_{43} \mu+2 j_{53}\right)}{j_{53} \mu} & j_{43} \\
j_{51} & j_{52} & j_{53} \\
j_{61} & 0 & 0
\end{array}\right], \operatorname{det}(j)=2 \frac{j_{61} j_{52} j_{53}}{\mu}\right]} \\
{\left[\lambda \neq 0, \lambda=-1, \eta=\frac{j_{43} \mu+j_{53}}{j_{53}}, \nu=-\frac{\left(j_{43} \mu+2 j_{53}\right) j_{43}}{j_{53}{ }^{2}}, \mu=\mu\right]}
\end{array}\right] \\
& \left.\begin{array}{c}
{\left[j==\left[\begin{array}{ccc}
j_{41} & j_{42} & j_{43} \\
j_{51} & j_{52} & 0 \\
j_{61} & 0 & 0
\end{array}\right], \operatorname{det}(j)=-j_{43} j_{52} j_{61}\right]} \\
{\left[\lambda \neq 0, \lambda=-1, \eta=-1, \nu=2 \frac{j_{42}}{j_{52}}, \mu=0\right]}
\end{array}\right]
\end{aligned}
$$

(b) For a type (ii) representation: there are 11 solutions.

$$
\begin{aligned}
& \begin{array}{c}
{\left[j==\left[\begin{array}{ccc}
j_{41} & j_{42} & -\frac{j_{53} \nu}{\eta} \\
j_{51} & -\frac{j_{42}(\eta-1)}{\nu} & j_{53} \\
j_{61} & j_{62} & 0
\end{array}\right], \operatorname{det}(j)=-\frac{j_{53}\left(\eta j_{41} j_{62}+j_{51} j_{62} \nu-j_{42} j_{61}\right)}{\eta}\right]} \\
{\left[\lambda=0, \eta=\eta, \nu=\nu, \mu=-\frac{\eta(\eta-1)}{\nu}, \varepsilon=0\right]}
\end{array} \\
& \begin{array}{c}
{\left[j==\left[\begin{array}{ccc}
j_{41} & j_{42} & 0 \\
j_{51} & \mu j_{42} & j_{53} \\
j_{61} & j_{62} & 0
\end{array}\right], \operatorname{det}(j)=-j_{41} j_{53} j_{62}+j_{42} j_{53} j_{61}\right]} \\
{[\lambda=0, \eta=1, \nu=0, \mu=\mu, \varepsilon=0]}
\end{array} \\
& \begin{array}{c}
{\left[j==\left[\begin{array}{ccc}
j_{41} & \nu j_{52} & j_{43} \\
j_{51} & j_{52} & 0 \\
j_{61} & j_{62} & 0
\end{array}\right], \operatorname{det}(j)=j_{43} j_{51} j_{62}-j_{43} j_{52} j_{61}\right]} \\
{[\lambda=0, \eta=0, \nu=\nu, \mu=0, \varepsilon=0]}
\end{array} \\
& \begin{array}{c}
{\left[j==\left[\begin{array}{ccc}
j_{41} & 0 & j_{43} \\
j_{51} & j_{52} & -\mu j_{43} \\
j_{61} & j_{62} & 0
\end{array}\right], \operatorname{det}(j)=j_{41} \mu j_{43} j_{62}+j_{43} j_{51} j_{62}-j_{43} j_{52} j_{61}\right]} \\
{[\lambda=0, \eta=0, \nu=0, \mu=\mu, \varepsilon=0]}
\end{array}
\end{aligned}
$$




$$
\begin{aligned}
& \left.\begin{array}{c}
{\left[j==\left[\begin{array}{ccc}
j_{41} & 0 & j_{43} \\
j_{51} & j_{52} & j_{53} \\
j_{61} & 0 & 0
\end{array}\right], \operatorname{det}(j)=-j_{43} j_{52} j_{61}\right]} \\
{\left[\lambda=0, \eta=0, \nu=0, \mu=-\frac{j_{53}}{j_{43}}, \varepsilon=0\right]}
\end{array}\right] \\
& \left.\begin{array}{c}
{\left[j==\left[\begin{array}{ccc}
j_{41} & j_{42} & j_{43} \\
j_{51} & -\frac{(\eta-1) j_{42}}{\nu} & -\frac{j_{43}(\eta-1)}{\nu} \\
j_{61} & j_{62} & j_{63}
\end{array}\right], \operatorname{det}(j)=-\frac{\left(j_{42} j_{63}-j_{43} j_{62}\right)\left(\eta j_{41}+j_{51} \nu-j_{41}\right)}{\nu}\right]} \\
{\left[\lambda \neq 0, \lambda=1, \eta=\eta, \nu=\nu, \mu=-\frac{\eta(\eta-1)}{\nu}\right]}
\end{array}\right] \\
& \begin{array}{c}
{\left[j==\left[\begin{array}{ccc}
j_{41} & j_{42} & j_{43} \\
j_{51} & \mu j_{42} & \mu j_{43} \\
j_{61} & j_{62} & j_{63}
\end{array}\right], \operatorname{det}(j)=j_{41} \mu j_{42} j_{63}-j_{41} \mu j_{43} j_{62}-j_{42} j_{51} j_{63}+j_{43} j_{51} j_{62}\right]} \\
{[\lambda \neq 0, \lambda=1, \eta=1, \nu=0, \mu=\mu]}
\end{array} \\
& \begin{array}{c}
{\left[j==\left[\begin{array}{ccc}
j_{41} & j_{52} \nu & j_{53} \nu \\
j_{51} & j_{52} & j_{53} \\
j_{61} & j_{62} & j_{63}
\end{array}\right], \operatorname{det}(j)=-j_{52} \nu j_{51} j_{63}+j_{53} \nu j_{51} j_{62}+j_{41} j_{52} j_{63}-j_{41} j_{53} j_{62}\right]} \\
{[\lambda \neq 0, \lambda=1, \eta=0, \nu=\nu, \mu=0]}
\end{array} \\
& \begin{array}{c}
{\left[j==\left[\begin{array}{ccc}
j_{41} & 0 & 0 \\
j_{51} & j_{52} & j_{53} \\
j_{61} & j_{62} & j_{63}
\end{array}\right], \operatorname{det}(j)=j_{41}\left(j_{52} j_{63}-j_{53} j_{62}\right)\right]} \\
{[\lambda \neq 0, \lambda=1, \eta=0, \nu=0, \mu=\mu]}
\end{array} \\
& {\left[\begin{array}{c}
{\left[j==\left[\begin{array}{ccc}
j_{41} & 0 & j_{43} \\
j_{51} & 0 & j_{53} \\
j_{61} & j_{62} & 0
\end{array}\right], \operatorname{det}(j)=-j_{41} j_{53} j_{62}+j_{43} j_{51} j_{62}\right]} \\
{\left[\lambda \neq 0, \lambda=\lambda, \eta=\frac{\mu j_{43}-j_{53} \lambda+j_{53}}{j_{53}}, \nu=-\frac{j_{43}\left(\mu j_{43}-2 j_{53} \lambda+j_{53}\right)}{j_{53}{ }^{2}}, \mu=\mu\right]}
\end{array}\right]} \\
& \left.\begin{array}{c}
{\left[j==\left[\begin{array}{ccc}
j_{41} & 0 & j_{43} \\
j_{51} & 0 & 0 \\
j_{61} & j_{62} & 0
\end{array}\right], \operatorname{det}(j)=j_{43} j_{51} j_{62}\right]} \\
{[\lambda \neq 0, \lambda=\lambda, \eta=\lambda, \nu=\nu, \mu=0]}
\end{array}\right]
\end{aligned}
$$

(4) CASE IV: $\mathfrak{k}=\mathfrak{r}_{3, \delta}$.

(a) For a type (i) representation: there are 6 solutions.

$$
\left.\begin{array}{c}
{\left[j==\left[\begin{array}{ccc}
j_{41} & i j_{43} & j_{43} \\
j_{51} & 0 & 0 \\
j_{61} & -i j_{63} & j_{63}
\end{array}\right], \operatorname{det}(j)=-2 i j_{51} j_{43} j_{63}\right]} \\
{[\delta=i, \eta=2, \nu=i, \mu=0]}
\end{array}\right]
$$




$$
\begin{aligned}
& \begin{array}{c}
{\left[j==\left[\begin{array}{ccc}
j_{41} & i j_{43} & j_{43} \\
j_{51} & i j_{53} & j_{53} \\
j_{61} & -i j_{63} & j_{63}
\end{array}\right], \operatorname{det}(j)=2 i j_{41} j_{53} j_{63}-2 i j_{51} j_{43} j_{63}\right]} \\
{[\delta=i, \eta=-2, \nu=i, \mu=0]}
\end{array} \\
& \begin{array}{c}
{\left[j==\left[\begin{array}{ccc}
j_{41} & -i j_{43} & j_{43} \\
j_{51} & -i j_{53} & j_{53} \\
j_{61} & i j_{63} & j_{63}
\end{array}\right], \operatorname{det}(j)=-2 i j_{41} j_{53} j_{63}+2 i j_{51} j_{43} j_{63}\right]} \\
{[\delta=-i, \eta=-2, \nu=-i, \mu=0]}
\end{array} \\
& \begin{array}{c}
{\left[j==\left[\begin{array}{ccc}
j_{41} & \frac{i j_{53}(\eta+2)}{\mu} & \frac{j_{53}(\eta+2)}{\mu} \\
j_{51} & i j_{53} & j_{53} \\
j_{61} & -i j_{63} & j_{63}
\end{array}\right], \operatorname{det}(j)=\frac{-2 i j_{53} j_{63}\left(\eta j_{51}-\mu j_{41}+2 j_{51}\right)}{\mu}\right]} \\
{[\delta=i, \eta=\eta, \nu=i, \mu=\mu]}
\end{array} \\
& \begin{array}{c}
{\left[j==\left[\begin{array}{ccc}
j_{41} & \frac{-i j_{53}(\eta+2)}{\mu} & \frac{j_{53}(\eta+2)}{\mu} \\
j_{51} & -i j_{53} & j_{53} \\
j_{61} & i j_{63} & j_{63}
\end{array}\right], \operatorname{det}(j)=\frac{2 i j_{53} j_{63}\left(\eta j_{51}-\mu j_{41}+2 j_{51}\right)}{\mu}\right]} \\
{[\delta=-i, \eta=\eta, \nu=-i, \mu=\mu]}
\end{array}
\end{aligned}
$$

(b) For a type (ii) representation: there are 4 solutions.

$$
\left.\begin{array}{c}
{\left[j==\left[\begin{array}{ccc}
j_{41} & j_{42} & j_{43} \\
j_{51} & -\frac{(\eta-1) j_{42}}{\nu} & -\frac{(\eta-1) j_{43}}{\nu} \\
j_{61} & j_{62} & j_{63}
\end{array}\right], \operatorname{det}(j)=-\frac{\left(j_{42} j_{63}-j_{43} j_{62}\right)\left(\eta j_{41}+\nu j_{51}-j_{41}\right)}{\nu}\right]} \\
{\left[j==\left[\begin{array}{ccc}
j_{41} & j_{42} & j_{43} \\
j_{51} & \mu j_{42} & \mu j_{43} \\
j_{61} & j_{62} & j_{63}
\end{array}\right], \operatorname{det}(j)=j_{41} \mu j_{42} j_{63}-j_{41} \mu j_{43} j_{62}-j_{42} j_{51} j_{63}+j_{43} j_{51} j_{62}\right]}
\end{array}\right]
$$

This gives the results in Table 2 .

\subsection{Tables.}




\begin{tabular}{|c|c|c|c|c|}
\hline & $\mathfrak{k}$ & Type $t$ & Existence results & Parameters of $t$ for the existence \\
\hline & $\mathfrak{h}_{1}$ & (i) & no & \\
\hline & & (ii) & no & \\
\hline & & (iii) & yes & $\nu=0$ \\
\hline & & (iv) & yes & $\eta=0$ \\
\hline & $\mathfrak{r}_{3}$ & (i) & no & \\
\hline & & (ii) & no & \\
\hline & & (iii) & yes & $\nu=1$ \\
\hline & & (iv) & yes & $\eta=1$ \\
\hline & $\begin{array}{l}\mathfrak{r}_{3, \lambda} \\
\lambda=0\end{array}$ & (i) & yes & $\begin{array}{c}\eta=\nu=0, \mu=1, \varepsilon=0 \\
\eta=\mu, \nu=1, \varepsilon=0 \\
\eta=1, \nu=\mu=0, \varepsilon=0 \\
\nu=1, \mu=0, \varepsilon=0 \\
\nu=0, \mu=1, \varepsilon=0 \\
\eta=1, \nu=0, \varepsilon=0 \\
\eta=1, \mu=0, \varepsilon=0 \\
\eta=0, \mu=1, \varepsilon=0 \\
\eta=0, \nu=1, \varepsilon=0 \\
\eta=, \mu=\nu=1 \varepsilon=0 \\
\eta=1, \nu=0, \mu=1, \varepsilon=0 \\
\eta=1, \nu=1, \mu=0, \varepsilon=0\end{array}$ \\
\hline & & (ii) & yes & $\begin{array}{l}\eta=1, \nu=\mu=\varepsilon=0 \\
\nu=1, \eta=\mu=\varepsilon=0\end{array}$ \\
\hline & & (iii) & yes & $\begin{array}{l}\eta=1, \nu=\varepsilon=0 \\
\nu=1, \eta=\varepsilon=0\end{array}$ \\
\hline & & (iv) & no & \\
\hline Table 1 & $\begin{array}{l}\mathfrak{r}_{3, \lambda} \\
\lambda \neq 0\end{array}$ & (i) & yes & $\begin{array}{c}\eta=1, \mu=\lambda \\
\eta=1, \nu=\lambda \\
\eta=\lambda, \nu=1 \\
\eta=\lambda, \mu=1 \\
\nu=1, \mu=\lambda \\
\nu=\lambda, \mu=1 \\
\eta=1, \nu=\mu=\lambda \\
\eta=\nu=1, \mu=\lambda \\
\eta=\mu=1, \nu=\lambda \\
\eta=\nu=\lambda, \mu=1 \\
\eta=\mu=\lambda, \nu=1 \\
\eta=\lambda, \nu=\mu=1 \\
\lambda=\eta=\nu=1 \\
\lambda=\eta=\mu=1 \\
\lambda=\nu=\mu=1 \\
\lambda=\eta=\nu=\mu=1\end{array}$ \\
\hline & & (ii) & yes & $\begin{array}{c}\lambda=\nu=1, \mu=0 \\
\eta=1, \nu=\lambda, \mu=0 \\
\eta=\lambda, \nu=1, \mu=0\end{array}$ \\
\hline & & (iii) & yes & $\begin{array}{c}\eta=1, \nu=\lambda \\
\eta=\lambda, \nu=1 \\
\lambda=\eta=\nu=1\end{array}$ \\
\hline & & (iv) & no & \\
\hline & $\mathfrak{r}_{3, \delta}$ & (i) & yes & $\begin{array}{l}\delta=0, \nu=0, \mu=1 \\
\delta=0, \eta=1, \nu=0\end{array}$ \\
\hline & & (ii) & yes & $\begin{array}{c}\nu=1, \mu=\delta \\
\nu=1, \mu=-\delta\end{array}$ \\
\hline & & (iii) & yes & $\delta=0, \eta=\nu=1$ \\
\hline & & (iv) & no & \\
\hline
\end{tabular}




\begin{tabular}{|c|c|c|c|c|}
\hline \multirow{9}{*}{ Table 2} & $\mathfrak{k}$ & Type $t$ & Existence results & Parameters of $t$ for the existence \\
\hline & $\mathfrak{h}_{1}$ & (i) & yes & $\begin{array}{c}\nu \neq 0, \mu=-\frac{\eta^{2}}{\nu} \\
\eta=\nu=0\end{array}$ \\
\hline & & (ii) & no & \\
\hline & $\mathfrak{r}_{3}$ & (i) & no & \\
\hline & $\mathfrak{r}_{3}$ & (ii) & no & \\
\hline & $\mathfrak{r}_{3, \lambda}$ & (i) & yes & $\begin{array}{c}\lambda=0, \nu \neq 0, \varepsilon=0 \\
\lambda=0, \eta=1, \nu=0, \varepsilon=0 \\
\lambda=0, \eta=-1, \nu=0, \varepsilon=0 \\
\lambda=-1, \eta=1, \mu=0 \\
\lambda=-1, \eta=\frac{j_{43} \mu+j_{53}}{j_{53}^{2}}, \nu=-\frac{\left(j_{43} \mu+2 j_{53}\right) j_{43}}{j_{53}^{2}} \\
\lambda=-1, \eta=-1, \nu=2 \frac{j_{42}}{j_{52}}, \mu=0\end{array}$ \\
\hline & & (ii) & yes & $\begin{array}{c}\lambda=0, \nu \neq 0, \mu=-\frac{\eta(\eta-1)}{\nu}, \varepsilon=0 \\
\lambda=0, \eta=1, \nu=0, \varepsilon=0 \\
\lambda=0, \eta=\nu=0, \varepsilon=0 \\
\lambda=0, \eta=\mu=0, \varepsilon=0 \\
\lambda=0, \eta=\nu=0, \mu=-\frac{j_{53}}{j_{43}}, \varepsilon=0 \\
\lambda=1, \nu \neq 0, \mu=-\frac{\eta(\eta-1)}{\nu} \\
\lambda=1, \eta=1, \nu=0 \\
\lambda=1, \eta=\mu=0 \\
\lambda=1, \eta=\nu=0 \\
\lambda \neq 0, \eta=\frac{\mu j_{43}-\lambda j_{53}+j_{53}}{j_{53}}, \nu=-\frac{j_{43}\left(\mu j_{43}-2 \lambda j_{53}+j_{53}\right)}{j_{53}^{2}} \\
\lambda \neq 0, \eta=\lambda, \mu=0\end{array}$ \\
\hline & $\mathfrak{r}_{3, \delta}$ & (i) & no & \\
\hline & & (ii) & yes & $\begin{array}{c}\delta=0, \nu \neq 0, \mu=-\frac{\eta(\eta-1)}{\nu} \\
\delta=0, \eta=1, \nu=0 \\
\delta=0, \eta=\mu=0 \\
\delta=0, \eta=\nu=0\end{array}$ \\
\hline
\end{tabular}

\section{REFERENCES}

[1] A. Andrada, M. L. Barberis, I. G. Dotti, G. P. Ovando, Product structures on four dimensional solvable Lie algebras. Homology Homotopy and Applications 7, 9-37 (2005).

[2] J. Milnor, Curvatures of left invariant metrics on Lie groups, Advances in Mathematics, 21, 293-329 (1976).

[3] V. S. Varadarajan, Lie Groups, Lie Algebras and Their Representations, Springer-Verlag New York, Graduate Texts in Mathematics, 102, (1984).

R. Campoamor Stursberg: I.M.I and Depto. Geometra y Topologa, Universidad Complutense de MADRID, SPAIN

E-mail address: rutwig@ucm.es

I. E. Cardoso, ECEn-FCeia, Universidad Nacional de Rosario, Pellegrini 250, 2000 Rosario, Santa Fe, Argentina

E-mail address: isolda@fceia.unr.edu.ar

G. P. Ovando: COnicet and ECEn-FCeia, Universidad Nacional de Rosario, Pellegrini 250, 2000 Rosario, Santa Fe, Argentina

E-mail address: gabriela@fceia.unr.edu.ar 